\title{
MMTF: The Maryland-Magellan Tunable Filter
}

\author{
S. Veilleux ${ }^{1,2}$, B. J. Weiner ${ }^{1,3}$, D. S. N. Rupke ${ }^{1,4}$, M. McDonald ${ }^{1}$, C. Birk ${ }^{5}$, J. \\ Bland-Hawthorn ${ }^{6,7}$, A. Dressler ${ }^{5}$, T. Hare ${ }^{5}$, D. Osip ${ }^{8}$, C. Pietraszewski ${ }^{9}$, and S. N. Vogel ${ }^{1}$
}

\begin{abstract}
This paper describes the Maryland-Magellan Tunable Filter (MMTF) on the Magellan-Baade 6.5-meter telescope. MMTF is based on a 150-mm clear aperture Fabry-Perot (FP) etalon that operates in low orders and provides transmission bandpass and central wavelength adjustable from $\sim 5$ to $\sim 15 \AA$ and from $\sim 5000$ to over $\sim 9200 \AA$, respectively. It is installed in the Inamori Magellan Areal Camera and Spectrograph (IMACS) and delivers an image quality of $\sim 0^{\prime \prime} .5$ over a field of view of $27^{\prime}$ in diameter (monochromatic over $\sim 10^{\prime}$ ). This versatile and easyto-operate instrument has been used over the past three years for a wide variety of projects. This paper first reviews the basic principles of FP tunable filters, then provides a detailed description of the hardware and software associated with MMTF and the techniques developed to observe with this instrument and reduce the data. The main lessons learned in the course of the commissioning and implementation of MMTF are highlighted next, before concluding with a brief outlook on the future of MMTF and of similar facilities which are soon coming on line.
\end{abstract}

\footnotetext{
${ }^{1}$ Department of Astronomy, University of Maryland, College Park, MD 20742; veilleux@astro.umd.edu, mcdonald@astro.umd.edu, vogel@astro.umd.edu

${ }^{2}$ Also: Max-Planck-Institut für Extraterrestrische Physik, Postfach 1312, D-85741 Garching, Germany

${ }^{3}$ Current Address: Steward Observatory, University of Arizona, 933 N. Cherry Ave., Tucson, Arizona 85721; bjw@as.arizona.edu

${ }^{4}$ Current Address: Institute for Astronomy, University of Hawaii, 2680 Woodlawn Drive, Honolulu, HI 96822; drupke@ifa.hawaii.edu

${ }^{5}$ Observatories of the Carnegie Institution for Science, 813 Santa Barbara Street, Pasadena, CA 91101; birk@obs.carnegiescience.edu, dressler@obs.carnegiescience.edu, thare@obs.carnegiescience.edu

${ }^{6}$ School of Physics, University of Sydney, Sydney, NSW, Australia; jbh@physics.usyd.edu.au

${ }^{7}$ Anglo-Australian Observatory, P. O. Box 296, Epping, NSW 2121, Australia

${ }^{8}$ Las Campanas Observatory, Carnegie Observatories, Casilla 601, La Serena, Chile; dosip@lco.cl

${ }^{9}$ IC Optical Systems Ltd, 190-192 Ravenscroft Road, Beckenham, Kent BR3 4TW, United Kingdom; chris.pietraszewski@icopticalsystems.com
} 
Subject headings: instrumentation: interferometers - instrumentation: spectrographs — methods: data analysis — techniques: image processing — techniques: spectroscopic

\section{Introduction}

The science drivers for tunable narrowband imaging of the night sky are very strong: (1) isolate the faint signal (e.g. emission line signatures) from distant objects against the bright noise from the sky or the source itself (e.g. continuum emission); (2) achieve high survey efficiency by combining high throughput, large field of view (FOV) and good spectral resolution (Jacquinot 1954); (3) produce well-defined volume-limited samples of objects selected by the wavelength (redshift) coverage of the instrument and unaffected by the morphological (compact versus diffuse) and/or broadband brightness biases generally associated with multi-object spectroscopy. A judicious choice of emission lines also helps select targets by precisely the quantity that one tries to measure (e.g. H $\alpha$, [O II] $\lambda \lambda 3727$, or Ly $\alpha$ for the star formation rates in galaxies; Jones \& Bland-Hawthorn 2001).

The ideal tunable filter is an imaging device which can isolate an arbitrary spectral band $\delta \lambda$ at an arbitrary wavelength $\lambda$ over a broad, continuous spectral range, preferably with a response function which is identical in form at all wavelengths. As described in BlandHawthorn (2000a, 2000b), a rich variety of physical phenomena can isolate a finite spectral band: absorption, scattering, diffraction, evanescence, birefringence, acousto-optics, singlelayer and multilayer interference, multi-path interferometry, polarizability and so on. All spectroscopic techniques rely ultimately on the interference of beams that traverse different optical paths to form a signal. The technologies which come closest to the ideal tunable filter are the air-gap Fabry-Perot (FP) and Michelson (Fourier transform) interferometers.

Recent technological developments in the fabrication of high-performance FP etalons 1 have allowed the implementation of reliable and cost-effective FP tunable filters with wide monochromatic FOV and adjustable transmission characteristics. These low-order FP etalons are the most straightforward application of tunable filter technology. Other, more sophisticated techniques such as Lyot and acousto-optic filters exist, but all are currently consider-

\footnotetext{
1 Alignment between the two highly polished plates of glass in these etalons can be maintained down to spacings of $2 \mu \mathrm{m}$ (measured at the coating surfaces). Long-range stacked piezo-electric transducers (PZT) allow the parallel plates to be scanned over a physical spacing of $4 \mu \mathrm{m}$ (5 wavelengths or 10 interference orders in the I band). Reflective coatings are laid down with ionic bombardment which allows for very high integrity and uniformity in the coating response over a broad wavelength range.
} 
ably more expensive, more risky, and typically have lower throughput.

Deep optical emission-line surveys with the prototype FP tunable filter, the Taurus Tunable Filter (or TTF; Bland-Hawthorn \& Jones 1998a, 1998b; Bland-Hawthorn \& KedzioraChudczer 2003) on the $3.9 \mathrm{~m}$ Anglo-Australian Telescope, have shown that there is a rich field of science awaiting exploration with large ground-based telescopes equipped with these filters. In this paper, we describe the first such tunable filter in routine operation on a large telescope: the Maryland-Magellan Tunable Filter (MMTF) on the Magellan Baade 6.5-meter telescope at Las Campanas. MMTF was commissioned in June 2006 and has been used as a PI instrument since January 2007. A number of engineering runs over the past two years have allowed us to fine tune MMTF and make it easier to use. This versatile and easy-to-use instrument is currently being used for science at a rate of $\sim 10$ nights per semester.

In this paper, we summarize the technical aspects of MMTF, discussing them primarily from a user's point of view. In Section 2, we introduce the basic concepts behind FP tunable filters. In Sections 3 and 4, we describe the hardware and software associated with MMTF and the techniques used to observe with this instrument and reduce the data. In Section 5, we highlight the main lessons learned in the course of the commissioning and implementation of MMTF. In Section 6, we conclude with a discussion of the future of MMTF and of similar facilities which are coming on line in the near future. The official website of MMTF is http://www.astro.umd.edu/ veilleux/mmtf/ and should be consulted for more detail.

\section{Basic Principles of FP Tunable Filters}

FP tunable filters operate on similar principles to narrow-band interference filters. In a single-plate interference filter, the interference occurs in the interior of a solid plate, where the two sides of the plate act as reflective surfaces (e.g. MacLeod 2001). In a FP tunable filter, the interference arises in the air gap between the surfaces of two separate plates of high reflectivity. Light travels through the interferometer such that at each radius from the optical axis a specific wavelength is imaged, resulting in the wavelength, $\lambda$, varying linearly with the cosine of the angle from the optical axis, $\theta$ (At the detector, the $\theta$ dependence translates into a radial dependence of wavelength via the camera focal length; the optical axis is at the center of this radial pattern):

$$
m \lambda=2 \mu l \cos \theta
$$

where $m$ is the order number, $l$ is the spacing between the two plates, and $\mu$ is the index of refraction of the air gap. To first approximation, $\mu \approx 1$ and $\theta \approx 0$ so $l \approx m \lambda / 2$. Low-order etalons of tunable filters require small plate spacings of a few microns. 
From eqn. (11), assuming $\mu \simeq 1$ and $\theta \simeq 0$, one finds that interference orders are separated by a free spectral range, $F S R=\lambda / m \simeq \lambda^{2} / 2 l$. Each transmission peak is characterized by an Airy profile. The ratio of the free spectral range to the instrumental resolution is the effective finesse, $N$, or the number of independent resolution elements within one FSR. In a perfect system, the effective finesse is equal to the reflective finesse, $N_{R}$, which is only a function of the reflection coefficient, $\Re$, of the coatings: $N_{r}=\pi \Re^{1 / 2} /(1-\Re)$. However, irregularities in the plate surfaces and coating thicknesses, as well as deviations from perfectly parallel plates (e.g. bowing), degrade the effective finesse, and hence the efficiency of observing. The effective finesse is approximately given by $1 / N^{2}=1 / N_{r}^{2}+1 / N_{d}^{2}+1 / N_{a}^{2}$, where $N_{r}$ is the reflective finesse, $N_{d}$ is the defect finesse due to plate defects, and $N_{a}$ is the aperture finesse due to the solid angle of the beam (e.g. Atherton et al. 1981).

FP tunable filters work at low orders of interference to ensure that the wavelength varies little over the full FOV. In other words, they provide quasi-monochromatic images with a single exposure. The monochromatic, or Jacquinot, spot is defined to be the region over which the change in wavelength does not exceed $\sqrt{2}$ times the etalon bandpass, $\delta \lambda$. From eqn. (1), the relative change in wavelength $[\lambda(0)-\lambda(\theta)] / \lambda(0)$ as a function of off-axis angle $\theta$ is $1-\cos \theta \simeq \theta^{2} / 2$ so

$$
\frac{\theta_{\mathrm{mono}}^{2}}{2} \simeq \frac{\sqrt{2} \delta \lambda}{\lambda} .
$$

For a wavelength $\lambda$, the bandpass relates directly to the order $m$ such that $\delta \lambda=\lambda / R=$ $\lambda / N m$, where $R$ is the spectral resolving power and $N$ is the effective finesse (e.g. Bland \& Tully 1989). Combining this expression with eqn. (2) we find that the angle subtended by the monochromatic spot is

$$
\theta_{\text {mono }}^{2} \simeq 2 \sqrt{2} /(N m)
$$

For a given order (or, equivalently, via eqn. (11), a given plate spacing $l$ ), the angular size of the monochromatic spot thus depends on wavelength only through the finesse (the finesse depends on wavelength through the wavelength-dependent transmission of the plate coatings). Eqn. (3) shows how the spot covers increasingly larger areas on the detector as the filter is used at lower orders of interference.

Another notable advantage of FP tunable filters over other spectrographs is their ability to provide adjustable resolution. Since $R=N m$, the spectral resolving power, $R$, changes only slowly between orders when the order number, $m$, is large. One can only get a useful range of resolutions at low orders, precisely where FP tunable filters operate. 


\section{Description of MMTF}

\subsection{Host Instrument}

The etalon of MMTF is installed in the collimated beam of IMACS, the Inamori Magellan Areal Camera and Spectrograph (Dressler et al. 2006). IMACS mounts at the Nasmyth focus of the Magellan-Baade Telescope. Fed by the f/11 Gregorian configuration, with an integral atmospheric dispersion corrector (ADC) and field corrector mounted at the tertiary mirror, the transmitting, all-spherical collimator produces a well corrected, unvignetted field of $24^{\prime}$ in diameter, and slightly vignetted field of $30^{\prime}$ in diameter. Two cameras are used to re-image the $150-\mathrm{mm}$ diameter collimator exit pupil at $0^{\prime \prime} .111$ and 0.200 pixel $^{-1}$ on the detector. To avoid any major modifications to the design of IMACS and optimize the FOV, MMTF strictly utilizes the short focal length camera.

The optical path through IMACS and MMTF is shown in Figure 1. The etalon of MMTF sits in one of six interchangeable positions of the disperser server (Figure 2). A mounting plate was fabricated to mate the Fabry-Perot etalon to the disperser server and facilitate installation and removal of MMTF. The CCD array in the $\mathrm{f} / 2$ configuration, Mosaic2, consists of a mosaic of $8 \mathrm{E} 2 \mathrm{~V} 2048 \times 4096$ devices with $15 \mu \mathrm{m}$ pixels.

The MMTF control software is fully integrated into the IMACS control software. This aspect of MMTF is described in Section 4 below.

\subsection{Main System Components}

The MMTF consists of 3 major components: the etalon and its support assembly, the CS100 controller, and the cables connecting them. The entire system, except the support assembly, was manufactured by IC Optical Systems Ltd.

The etalon, shown in Figure 2, consists of two parallel plates composed of fused silica, each with a 150-mm clear aperture. The etalons are separated by a sealed but tunable air gap. The plates are coated on the interior side (the side facing the other plate) with a reflective coating consisting of a multi-layer dielectric. The exterior surfaces are anti-reflection coated. The plates are smooth on small scale at the $\lambda / 100$ level. The etalon weight is $19.5 \mathrm{~kg}$, not including the mounting plate.

The reflection curve, $\Re(\lambda) \approx 1-T(\lambda)$ (neglecting absorption and scattering), of a fused silica witness plate that is coated in proximity to the etalon plates coating is shown in Figure

3 (the etalon plates and witness are coated simultaneously in the coating chamber so the 
only differences between plates and witnesses are due to distribution in the chamber. This is stated as being $1 \%$ or less). The finesse derived from this reflection curve is the theoretical maximum finesse and is considerably higher than the effective full-beam finesse of the etalon measured at the telescope (see Sections 3.4 and 5.1).

The CS100 control system is a three-channel bridge system which uses capacitance micrometers and long-range stacked PZT actuators, incorporated into the MMTF, to monitor and correct errors in mirror alignment and spacing. Two channels (XY) control alignment and the third (Z) maintains spacing by referencing the cavity length-sensing capacitor to a high stability standard reference capacitor, also located in the MMTF. Because this is a closed-loop system, non-linearity and hysteresis in the PZT drive are eliminated entirely, as of course are drifts in cavity alignment and spacing. The CS100 servo system works on a nulling method: any imbalance in the capacitance bridge causing an error signal is corrected for by a change in piezo voltage and hence plate spacing until the error is nulled.

The coarse values for tilt and spacing $\left(X_{\text {coarse }}, Y_{\text {coarse }}, Z_{\text {coarse }}\right)$ are set using dials on the CS100 controller. The tilt and spacing can be changed in smaller increments using $X_{\text {fine }}, Y_{\text {fine }}$, and $Z_{\text {fine }}$ values. The fine values can be changed using either the CS100 dials or the IMACS control software (described in Section 4 below). Note that $(X, Y)_{\text {coarse }}$ and $(X, Y)_{\text {fine }}$ control the etalon alignment. However, the coarse spacing $Z_{\text {coarse }}$ controls the etalon bandpass, while the fine spacing $Z_{\text {fine }}$ governs the transmitted wavelength. The fine spacing can affect the bandpass, but only when changed by a large amount.

The etalon connects to the CS100 through shielded cabling that runs from IMACS to the cooled electronic rack on one side of the Nasmyth platform. Since IMACS rotates to compensate for field rotation of the alt-az mount, the cabling has to be made long enough to accommodate this rotation. A 3-meter cable connects the etalon to the IMACS bulkhead via an internal cable wrap. A 15-meter cable connects the bulkhead to the electronic rack via an external cable wrap (see Dressler et al. 2006 for more detail on the cable wraps in IMACS).

\subsection{Order-Blocking Filters}

A given plate spacing transmits multiple wavelengths at a single location, each corresponding to a different order (see eqn. (1)), so a blocking filter is required to select only one transmission order. The order-blocking filters for MMTF are located in the $\mathrm{f} / 2$ converging beam of IMACS between the camera and the CCD array (see Figure 1). The central wavelengths and approximate full-widths at half-maximum of the transmission profiles (cor- 
rected for the convergence of the IMACS f/2 beam) of the current set of filters are: $5102 / 150$, $5290 / 156,6399 / 206,6600 / 260,6815 / 216,7045 / 228,8149 / 133$, and 9163/318 $\AA$. These filters were selected to provide access to several emission-line diagnostics (e.g. slightly redshifted [O III] $\lambda 5007,[\mathrm{O} \mathrm{I}] \lambda \lambda 6300,6364, \mathrm{H} \alpha,[\mathrm{N} \mathrm{II}] \lambda \lambda 6548,6583,[\mathrm{~S} \mathrm{II}] \lambda 6716,6731$, and [S III] $\lambda 9069)$ and two key long-wavelength atmospheric $\mathrm{OH}$-free windows centered around $\sim 8150$ and $\sim 9150 \AA$.

\subsection{Characteristics of MMTF Etalon}

As mentioned in Section 3.2, accurate determination of the characteristics of the MMTF etalon requires full-aperture illumination of the etalon in the same configuration (collimated beam) as that used at the telescope. Table 1 lists a representative summary of on-site measurements obtained at four different wavelengths (through four different order-blocking filters). A closer look at the entries in this table reveals a number of interesting features.

The available full-width at half maximum $(F W H M)$ instrumental resolution at 6600 $\AA$ is $6-13 \AA$. (As discussed in Jones et al. 2002, the "effective bandpass" of FP tunable filters, or the integral of the profile divided by its peak, is $\pi / 2 \times F W H M$ rather than $1.06 \times$ $F W H M$ in case of a Gaussian because the instrumental profile is very close to a Lorentzian; see also Section 4.3.5.) The values of $F W H M$ at other wavelengths, for a fixed plate spacing, scale roughly with $\lambda^{2}$ over $6600-9200 \AA$, as expected from the expresssion $F W H M=$ $F S R / N \simeq \lambda^{2} /[2 N l]$, where the effective finesse $N$ is mostly constant, except at the edges of the etalon's coating response. At the edges of the coating transmission curve (see Figure 3), the reflectivity (and hence the etalon finesse) drops, broadening the profile and increasing $F W H M$. Thus, at $5100 \AA, F W H M$ ranges from 8 to $14 \AA$. These values are slightly higher than at $6600 \AA$, while one would expect lower values for a constant finesse. The effective finesse is $\sim 24-29$ from $\sim 5300$ to $9200 \AA$, but only $\sim 13-14$ below $\sim 5300 \AA$ (column (6) in Table 1).

Note that the plate spacings listed in Table 1 represent the effective distances separating the reflective surfaces, taking into account the finite thickness of the coatings. It is derived empirically from $l=\lambda^{2} /[2 F S R]$. For a fixed physical gap (no change in $Z_{\text {coarse }}$ and $Z_{\text {fine }}$ ), we find that the effective spacing depends on wavelength, increasing beyond $6500 \AA$ (this wavelength dependence is also seen in the effective alignment of the plates; see Sections 4.3.1 and 5.1). 


\subsection{Monochromatic Spot Size}

As described in Section 2 (eqn. (3) ), for a given order, the area of approximately constant wavelength at the image center, or monochromatic spot, depends on wavelength only through the finesse. However, the diameter of the spot does depend on plate spacing via the order number. The maximum angle of the IMACS collimated beam is $\sim 10^{\circ}$ at the edge of the short camera field ( $F \simeq 365 \mathrm{~mm}$ at $\sim 6600 \AA$ with a tendency to increase at longer wavelengths; see Section 5.1 for more detail). This is to be compared with the size of the monochromatic spot derived from eqn. (3). Table 2 lists representative values for several different wavelengths and spacings. Notice the larger monochromatic spot at $5100 \AA$ due to the lower finesse at these wavelengths (Figure 3 and Table 1).

\subsection{Sensitivities and Throughput}

The overall sensitivities and throughput of MMTF were calculated at various wavelengths using continuum spectrophotometric flux standards (stars from Oke 1990 and Hamuy et al. 1992, 1994) and emission-line flux standards (planetary nebulae from Dopita \& Hua 1997). The procedure used to flux calibrate MMTF data is described in Section 4.3.5. The results are listed in Table 3. In columns (2) - (4), we list the the flux of a 5- $\sigma$ emission-line point source in a 1-hour exposure at New Moon, First or Third Quarter, and Full Moon, assuming $0.5^{\prime \prime}$ seeing, a $1^{\prime \prime}$ diameter extraction aperture, and an airmass of 1.1 . In column (5), we list the throughput in terms of the ratio of the CCD count rate to the flux. These measurements are for optimal plate alignment and assume that the source is a point source with an emission line centered in the bandpass. For a continuum source, the flux density in erg s${ }^{-1} \mathrm{~cm}^{-2} \AA^{-1}$ can be inferred by dividing the flux in $\mathrm{erg} \mathrm{s}^{-1} \mathrm{~cm}^{-2}$ by the effective etalon bandpass i.e. $\pi / 2 \times F W H M$ in $\AA$ (see Section 4.3 .5 for more detail; the values of $F W H M$ are given in Table 1).

\section{Using MMTF}

MMTF has been used for a wide range of applications, including (but not limited to) the following: imaging of Galactic line-emitting nebulae and stars, emission-line ratio ("excitation") maps of galaxies, searches for warm, diffuse gas on the outskirts of galaxies, suppression of quasar light in damped Ly $\alpha$ systems, wide-field surveys for high-redshift lineemitting galaxies. In this section, we discuss the technical aspects involved in the use of MMTF. 


\subsection{Modes of Operation}

MMTF operates in one of three modes: (1) staring mode, (2) wavelength scanning mode, and (3) charge shuffling and frequency switching (CS/FS) mode.

In the staring mode, the etalon gap is fixed and the result is a narrow-band image over the entire FOV. However, the wavelength varies slowly with distance from the optical axis (eqn. (11)).

In the scanning mode, an image is taken at a series of different etalon spacings (wavelength slices). The result is a low-resolution spectrum at each position. This mode is particularly useful to capture all line emission from a target which covers a range in velocity or is larger than the monochromatic spot.

The CS/FS mode enables the observer to switch among 2-3 central wavelengths during a single exposure. The procedure is as follows (see Figure 4): Each IMACS CCD is first divided into three equal segments by an aperture mask. The aperture allows light only onto the center third of each chip ("open" segments in Figure 4). When the exposure begins, the center of each chip is exposed at a single wavelength. With the shutter closed, the accumulated charge is then (almost instantaneously) moved down one-third of the chip length. The etalon's central wavelength is changed (again, almost instantaneously). The exposure proceeds at this second wavelength, again at the center of the chip, for a time equal to twice that at the first wavelength. The charge is then shuffled back up, and integration proceeds at a third wavelength atop the accumulated charge from the first part of the exposure for a time equal to that at the first wavelength. The procedure is then repeated. At the end of the exposure, each chip contains two images of the same field, each on one-third of the chip. The last third is empty. The third wavelength may equal the first, to produce two monochromatic images. Alternatively, it may be unique from the other two. In this case one of the resulting images is the sum of images at two separate wavelengths. Each wavelength in this image experiences one-half the exposure time of the other image.

The CS/FS procedure allows significant improvements in relative photometry. Time variations in transparency and seeing are matched between images at two different wavelengths, using a cadence chosen by the observer. A second advantage of charge-shuffling is that it allows accurate matching in wavelength space of the continuum of an emission-line source. If one image traces an emission-line, the other can trace the continuum at one or two immediately adjacent wavelengths. The time overhead for charge shuffling and changing wavelengths is limited by the shuffle time: $\sim 2.5$ msec per line, i.e. $\sim 3$ sec for 1300 lines. In comparison the shutter takes less than 1 second to open or close and the etalon response time is negligible. The shutter is closed before the charge is moved on the chip, and re-opened 
after the shuffle. The exposure time between charge shuffles is left up to the observer. Only $1 / 3$ of the FOV is illuminated during a given exposure. Thus, for observations of sources or fields covering the FOV, the camera must be dithered at least twice on $5^{\prime}$ scales to fill in the gaps. The voltage applied to the CCD chips for charge shuffling is different than for normal operation. This results in saturation effects when bright sources are in the field. Fields containing very bright stars should therefore be avoided.

\subsection{Installation, Startup, Takedown}

IMACS, the host instrument for MMTF, is by far the most popular instrument on Magellan-Baade. The MMTF etalon thus cannot stay in the IMACS disperser wheel when not in use. Although MMTF nights are generally scheduled in blocks to reduce the number of instrument switches per semester, an important operational requirement of MMTF was that installation, startup, and takedown be as straightforward as possible. At present, the installation, recabling, and startup procedure takes about an hour from start to finish. The etalon is left in its mounting plate between the runs to reduce overheads. The CS100 settings needed to balance the capacitance bridges of the etalon 2 and align the etalon plates have proven very stable from run to run. These settings are used as starting points for the final fine tuning of the alignment from the control room.

Remote computer control of the CS100 fine settings is done via the IMACS control software (coarse settings can only be changed manually). Release and re-acquisition of remote control on the CS100 is done via a button within a password-protected "HardHat" engineering GUI. Control of the CS100 is returned to the instrument front panel if, e.g., the etalon goes out of balance, $Z_{\text {coarse }}$ (the bandpass) needs to be changed, or the CS100 is being turned off at the end of a run.

MMTF observing is done using the standard IMACS data acquisition software along with a set of special built-in procedures. These include the ability to set the etalon fine X, Y, and $\mathrm{Z}$ values in the Hardhat GUI, as well as a set of programmable scripts which coordinate commands to the CCD camera and the CS100 for the following calibration and observation tasks: (1) wavelength scanning; (2) CS/FS; (3) determining the plate alignment; (4) taking "data sausages" for wavelength calibration. We explain these tasks in the following two sections, starting with the calibration tasks followed by the observation tasks.

\footnotetext{
2 The bridges can be unbalanced in several ways. For instance, temperature gradients across the MMTF cause changes in relative PZT lengths which cause error signals which are nulled by changes in the high voltage across the relevant PZT.
} 


\subsection{Calibrations}

Full calibration of MMTF consists of 5 different tasks: (1) aligning the etalon plates, (2) checking the position of the optical axis, (3) determining the wavelength calibration, (4) flat fielding the data, and (5) deriving absolute photometry. The first four of these can be carried out by the instrument scientist and/or the observer before the night begins. These tasks are carried out within the IMACS control software by creating standard scripts and running them.

\subsubsection{Plate Alignment}

Alignment is the process by which the two etalon plates are made parallel to one another. If the plates are not optimally aligned, the transmission profile will be broad and/or asymmetric. This diminishes the light transmitted in the core of the profile, and thus the system throughput (for an emission-line source) and the instrumental resolution. It is thus crucial that proper alignment is achieved and maintained throughout the run.

There are several possible methods for achieving parallel plates, providing various degree of accuracy (e.g. Jones \& Bland-Hawthorn 1998). We have developed a simple and efficient procedure which relies on scanning the transmitted image in the radial coordinate to produce a spectrum. The plate alignment is scanned along both axes of movement using a $(\mathrm{M} \times \mathrm{N})$ grid of $\left[X_{\text {fine }}, Y_{\text {fine }}\right]$. At each value of $\left[X_{\text {fine }}, Y_{\text {fine }}\right]$, an 1-5 sec exposure is taken of a bright emission line from an arc lamp appropriate for the order-blocking filter being used (Argon, Krypton, Neon, or Xenon). Each image is then azimuthally averaged to create an emissionline spectrum (Figure 5; see Section 4.5.3 for more detail on the method used to create this spectrum). The profiles of emission features are compared by eye (and, where possible, with line fits) to find the narrowest and most symmetric profile. An example of output from this procedure is shown in Figure 6 . This alignment procedure typically takes $\sim 15$ minutes from beginning to end.

As briefly described in Section 3.2, plate alignment is achieved and maintained through a capacitor and piezo-electric feedback system. However, we discovered that plate alignment unexpectedly depends on two variables: (1) the wavelength, due to changes in the behavior of the multi-layer reflection coating with wavelength; and (2) the rotation angle of the etalon, due to gravity-induced sag of the plates that remains uncorrected by the system feedback mechanism. These dependencies are shown in Figures 7 and 8, respectively. Alignment

changes by $\lesssim 0.02 \mu \mathrm{m}$ from 5100 to $6500 \AA$ but by $\sim 0.10-0.15 \mu \mathrm{m}$ from 6500 to $9100 \AA$. The total amplitude of the change in alignment with gravity angle is $\sim 0.03 \mu \mathrm{m}$, but is generally 
highly reproducible 3

The plate alignment therefore has to be adjusted whenever the order-blocking filter is changed. The change in alignment due to rotation is minimized by breaking up long exposures into segments of $\sim 20-30$ minutes and starting each exposure at approximately the same IMACS rotation angle (gravity angle). During each exposure, the IMACS camera rotates to maintain a constant orientation of the CCD mosaic with respect to the sky, but the change in gravity angle over the course of these short exposures is small enough to have no appreciable effect on plate alignment (this issue is discussed further in Sections 4.4 and $5.1)$.

\subsubsection{Optical Axis}

There is a wavelength gradient in the MMTF due to the angle at which rays pass through the etalon (eqn. (11)). This gradient is circularly symmetric about the optical axis (the projection of the MMTF normal axis onto the CCD). The optical axis is in the center of the FOV, but shifts slightly from run to run (due to the re-mounting of the IMACS CCD) and must be re-measured. During a particular run, it is constant within a few pixels as long as the etalon plates stay aligned.

To find the optical axis, we use the fact that there is a faint $(\sim 0.5 \%)$ ghost reflection between the MMTF and the CCD (this is a well-understood feature of FPs; Bland \& Tully 1989). Once the etalon plates are properly parallel, we insert into the beam a slit mask that has 5-10 guide star holes (square apertures) dispersed somewhat evenly over the central half of the FOV, and then take a quartz lamp exposure. The exposure must be long enough that faint reflections (primary ghost images) of the guide star boxes appear, but short enough that the original images do not saturate. These reflections are symmetric about the optical axis. An IRAF procedure is run to determine the positions of the guide star boxes and their reflections and then derive the location of the optical axis, typically with accuracy of $\lesssim 1$ pixel $\left(\lesssim 0^{\prime \prime} 2\right)$. An example of output from this procedure is shown in Figure 9 . This procedure typically takes $\sim 15$ minutes from beginning to end.

\footnotetext{
${ }^{3}$ For reasons which remain unclear, the alignment values become difficult to predict for gravity angles between -120 and -20 degrees, a region dubbed the "danger zone".
} 


\subsubsection{Wavelength Calibration}

On the optical axis, for effective plate spacing $l, \lambda=A+B Z_{\text {fine }}$, where $Z_{\text {fine }}=Z(l)$ represents voltages applied to the etalon by the CS100 and is the variable read by the IMACS

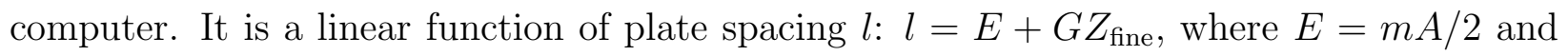
$G=m B / 2$. $A$ and $B$ thus vary from order to order. By substituting for $m$ using the interference equation, eqn. (1), we also see that $A$ and $B$ vary linearly with wavelength for a given plate spacing. More generally, using eqn. (11) at an arbitrary radius $R$ off the optical axis:

$$
\lambda(R)=\lambda(0) / \sqrt{\left(1+R^{2} / F^{2}\right)}=\left(A+B Z_{\text {fine }}\right) / \sqrt{\left(1+R^{2} / F^{2}\right)},
$$

where $F$ is the focal length of the camera (note that the wavelength decreases with increasing distance from the optical axis.).

Wavelength calibration involves finding $A$ (the wavelength scale zero point) and $B$ (三 $d \lambda / d Z$ ) in eqn. (4). A may vary with time (possibly due to temperature changes) and with the alignment setting. It is calibrated at the beginning of the run and checked frequently using single, full-field images of emission-line rings from an arc lamp (Figures 5 and 10; also see Section 4.4 for a discussion of $A$ drift monitoring). $B$ depends on the coarse plate spacing, $Z_{\text {coarse }}$, and the interference order. It is stable and has been measured for 3 values of $Z_{\text {coarse }}$ in each filter based on MMTF arc lamp spectra (Figure 11). These were extracted from "data sausages" (i.e. data cubes which are narrow in the X and Y directions but highly elongated in the $\mathrm{Z}$ direction). These sausages were obtained by scanning the etalon spacing through somewhat more than one free spectral range and using small CCD subrasters at 8 different distances from the optical axis (one on each CCD chip), including one near the center where the main science target is often positioned. It currently takes about 20-30 minutes to acquire these Nyquist-sampled data sausages. These spectra are compared with fully calibrated archived spectra obtained with the same settings to help with line and order identifications. The fit takes into account all eight data sausages and provide the $A$ and $B$ parameters for all orders that were sampled.

\subsubsection{Flat Fielding}

Flat fielding corrects for large-scale and pixel-to-pixel variations in sensitivity across the field. The amplitudes of these variations depend on wavelength so a series of flats should in principle be taken at each wavelength in which the science targets were observed. In practice, we have found that steps of $\sim 10 \AA$ are sufficient to correct for this wavelength dependence. A screen in front of the secondary mirror is illuminated by a bright quartz 
lamp and exposure times of 3-5 seconds are sufficient to get $\sim 1 \%$ accuracy. In the case of observations taken in the CS/FS mode (Section 4.1), the flats are taken in the exact same mode, adjusting the exposure times appropriately to prevent saturation.

\subsubsection{Absolute Photometry}

Absolute flux calibration with MMTF is straightforward. A spectrophotometric standard of moderate brightness (e.g. Oke 1990; Hamuy et al. 1992, 1994) is observed at roughly the same wavelength at which the science data were taken. The calibration star is positioned roughly at the same location as the science target, since wavelength changes with distance from the optical axis. The transmitted stellar flux from the standard is $f_{\lambda} \times$ "effective bandpass", where $f_{\lambda}$ is the continuum flux density of the star at wavelength $\lambda$ and the effective bandpass is the integral of the profile divided by its peak. As discussed in Section 3.4, the effective bandpass for the Lorentzian instrumental profile of MMTF is $\pi / 2 \times F W H M$ rather than $1.06 \times F W H M$ in case of a Gaussian (see Jones et al. 2002 for more detail).

Care must be exercised in choosing a stellar flux standard where the wavelengths of interest are not affected by stellar absorption lines (e.g. H $\alpha$ ). To avoid this problem altogether, emission-line flux standards (e.g. planetary nebulae of Dopita \& Hua 1997) are often used when observing in the $6600 \AA$ and $6815 \AA$ order-blocking filters. These emission-line stardards are observed in wavelength scanning mode (see Section 4.1 for a description) to produce integrated emission-line spectrum that can be directly compared with the known $\mathrm{H} \alpha,[\mathrm{N} \mathrm{II}] \lambda \lambda 6548,6583$ or [S II] $\lambda \lambda 6716,6731$ fluxes of these objects.

\subsection{Observations}

\subsubsection{Before the Run}

Three options are available to allow the observers to customize MMTF to their science program:

1. Choice of observing mode. As described in Section 4.1, MMTF can be used in three different modes. The staring mode is ideal for blind searches of emission-line sources over large areas. The scanning mode is useful to increase the volume of stare-mode blind searches or to study sources with a range of velocities or of angular size larger than the monochromatic spot. Finally, the CF/FS mode is well suited for high-precision differential experiments which require only a small fraction of the total FOV. 
2. Choice of spectral resolution. The choice of $Z_{\text {coarse, }}$ or coarse plate spacing, is a compromise between the size of the monochromatic spot and the signal-to-sky contrast. Larger values of $Z_{\text {coarse }}$ (higher orders) yield smaller monochromatic spot sizes (eqn. (3)). However, they also have smaller bandpasses, which means that the amount of sky emission transmitted is lower, thus increasing the signal-to-noise ratio. Three $Z_{\text {coarse }}$ settings are available to the observer; it is generally not changed during an observer's run due to overheads associated with the calibrations.

3. Choice of wavelength and order-blocking filters. The observer is limited to the use of at most 2 filters in a single night because of calibration overheads. Observers have full freedom to tune the central wavelength of the MMTF bandpass within the range allowed by a particular blocking filter. The wavelength can be tuned from one exposure to the next, or within a given exposure (see Section 4.1). Changing filters between exposures is straightforward. However, each filter requires its own set of calibrations to determine the wavelength solution and etalon alignment (Section 4.3).

The MMTF website has a number of GUI tools to help with proposal writing, run planning, and the observing itself: (1) a transmission calculator is available to determine if the wavelength of a particular redshifted feature falls within the order-blocking filters of MMTF and the corresponding transmission of the relevant filter. (2) a exposure time calculator has recently been implemented to determine the exposure time needed to reach a particular signal-to-noise ratio or, conversely, the signal-to-noise ratio reached for a particular exposure time. The wavelengths of strong $\mathrm{OH}$ lines that fall within the observed range are flagged to warn the users that the signal-to-noise ratio at these wavelengths will be degraded due to enhanced Poisson noise and larger systematic errors resulting from sky subtraction. (3) A wavelength calculator has been designed to help determine the exact position of emission lines on the detectors for a particular MMTF etalon setting based on a previously determined wavelength calibration. This last calculator is also used during the night to update the $A$ value of the wavelength calibration in case of drifts.

\subsubsection{During the Afternoon}

For each filter to be used during the night, the following steps are taken in the afternoon preceding the night: (1) aligning the etalon plates using the profiles of an emission line from an arc lamp (Section 4.3.1), (2) capture a binned reference image of an emission-line ring from an arc lamp (Section 4.3.3), and (3) take the data sausages of an arc lamp over more than one FSR (Section 4.3.3). These calibrations are generally performed at a gravity angle of $\theta_{\text {grav }} \sim 0^{\circ}$, the parked position of IMACS during the day. The results from the wavelength 
calibration $(A, B)$ are inserted in the wavelength calculator for use throughout the night.

\subsubsection{At the Start of the Night}

The first target is acquired at the same IMACS gravity angle as the calibrations to avoid shifts in plate alignment due to etalon rotation. Before the observation, an exposure is taken of an arc lamp using the same $Z_{\text {fine }}$ setting and on-chip binning as those used for the reference image. This image is azimuthally averaged to create an emission-line spectrum which is then compared with the afternoon reference image to search for possible shifts in the zero point of the wavelength calibration. The value of $A$ in the wavelength calculator is updated accordingly. Using these updated numbers, the appropriate $Z_{\text {fine }}$ for the wavelength of interest is computed and the etalon is set at this value.

\subsubsection{Throughout the Night}

Before each exposure, IMACS is rotated to match the gravity angle of the calibrations. This procedure is repeated before each exposure of a sequence to minimize errors in the wavelength solution. We have found that this "fixed gravity angle procedure" cuts down on nightly overheads by up to $1-2$ hours.

A reference ring is taken regularly to check for change in peak position (wavelength zeropoint, A) and line profile (plate alignment). This is done every $\sim 20-30$ minutes while the temperature is changing quickly at the start of the night, or every hour when the temperature is stable. If the line profiles have degraded (less narrow and/or less symmetric), the alignment is recomputed using the procedure described in Section 4.3.1 and a reference ring is reacquired to update the value of $A$ and recalculate the value of $Z_{\text {fine }}$ which corresponds to the wavelength of interest (Figures 6 and 10).

We have found that realignment of the plates is generally not needed during the night if the fixed gravity angle observing procedure is used. This procedure does not allow for exposure times longer than 20-30 minutes. Nevertheless, each of these relatively short exposures is sky-noise limited so there is no loss of signal-to-noise ratio when the multiple exposures of a sequence are combined together.

Dithering of $30^{\prime \prime}$ or more in each direction is recommended to achieve a continuous image uninterrupted by features or chip gaps. Dithering is important for IMACS imaging in order to bridge the inter-chip gaps, which are $\sim 50$ pixels, or $10^{\prime \prime}$, on average. MMTF also introduces a set of reflections of the chip edges (of relative intensity of a few percent) which 
are not corrected by the flat fields. These reflections contaminate $\sim 10$ rows and/or columns of each chip.

\subsubsection{At the End of the Night}

Flats are obtained for each setting separated by at least $\sim 10 \AA$ observed during the night (Section 4.3.4). These flat field exposures have been found to be quite stable with time so they are acquired either in the morning after the observing or the afternoon prior the observing. A series of biases (0-second exposures) is obtained after the flats.

\subsection{MMTF Data Reduction Pipeline}

We have developed a collection of Fortran routines and Perl-driven IRAF scripts to calibrate the MMTF, subtract sky background from data, and create annular image masks. This software is permanently installed on the computers at Magellan, but is also publicly available on the MMTF website for the user's convenience when doing calibrations and data reduction elsewhere.

The main steps in the reduction pipeline are: (1) bias subtraction and flat fielding, (2) cosmic ray and bad pixel masking, (3) sky subtraction, (4) astrometry registration and image mosaicking, and (5) PSF-matching and stacking. The data from IMACS emerge as 8 individual files for each exposure, one file per chip. Steps 1-4 operate on these individual files, while Step 5 operates on the mosaic. It takes approximately one day to reduce a night of MMTF data on a $2.8 \mathrm{GHz}$ machine with $2 \mathrm{~Gb}$ of memory. The most time-consuming step is the cosmic ray removal. Each step is briefly described below.

\subsubsection{Bias Subtraction and Flat Fielding}

Bias subtraction and flat fielding of MMTF data are straightforward: The row overscan is subtracted from all files. The biases are combined. The combined bias is subtracted from each source and flat field file. The column overscan is subtracted. The flats are combined. Each flat is normalized so that the central region of the FOV has an average value near unity. The same normalization factor is applied to each chip. If specified, scattered light signatures are removed from the flat field. The source files are divided by the normalized flat field exposure. 


\subsubsection{Cosmic Ray and Bad Pixel Masking}

Cosmic ray removal is achieved in two stages. First, we attempt to remove cosmic rays for each image individually. This is done using Pieter van Dokkum's "LA Cosmic" task for IRAF (for details, see http://www.astro.yale.edu/dokkum/lacosmic/). This task is called from a script, which has switches to allow control of such things as the sky level and the threshold sigma for rejection. Generally, four iterations is enough to remove the majority of the cosmic rays in each field. Known bad pixel regions (e.g. bad columns) are appended to the cosmic ray masks. Although a very effective task, LA Cosmic tends to leave residual bright pixels unmasked (although very rarely does it miss a cosmic ray completely). This is

remedied by using the IRAF "crgrow" task later on to expand the masks by a single pixel in each direction (Section 4.5.4) and filter out any residual cosmic rays using the "imcombine" during the stacking procedure (Section 4.5.5).

\subsubsection{Sky Subtraction}

Sky subtraction is performed using the MMTF calibration software. Given the knowledge of the mapping of the CCD pixels to the focal plane and the location of the optical axis, the sky spectrum is azimuthally averaged. Sources and cosmic rays are filtered out using a biweight statistic. If necessary, median smoothing is employed to smooth out the sky spectrum. This results in a robust removal of the sky using a single command. This procedure runs without user interaction. On a $2.8 \mathrm{GHz}$ machine with $2 \mathrm{~Gb}$ of memory, the sky subtraction takes approximately 5-10 minutes per exposure (for unbinned data). If the optical axis is not accurate to within $\sim 20$ pixels or so, dipole residuals appear after sky subtraction, either in individual exposures or in a stack of several exposures with small dithers. If the etalon plates are properly parallel, the optical axis position derived from the method described in Section 4.3 .2 will be correct well below this level. For exposures with large, extended sources in the FOV, a subset of the CCDs is selected to avoid bright source emission in the calculation of the sky spectrum.

\subsubsection{Astrometry Registration and Image Mosaicking}

Perhaps the most challenging aspect of IMACS data reduction is determining the astrometric solution that maps the physical CCD coordinates to locations on the sky. Because of the small focal ratio of the IMACS camera (f/2) when the MMTF is in use, as well as the wide FOV, significant deviations from a linear mapping occur. The higher-order poly- 
nomial terms of the FOV-to-sky mapping are known from observations of dense star fields. The script applies the World Coordinate System (WCS) information to all 8 chips for each exposure. This information contains the high-order distortions in the chips as well as the accurate chip spacings and the rotation and scale of the FOV. This information is used to properly register each FOV.

Cosmic ray masks are extended by one pixel in each direction to prevent ringing caused by residual cosmic rays in the interpolation steps. Masked pixels in the input files are replaced by 0.0 (the sky value) to prevent blurring of cosmic rays. These values will not play a role in the final image, since the contribution from masked images will be ignored. The only reason for this coarse interpolation is to prevent ringing in the next step.

The IRAF task "mscred.mscimage" is run on each individual chip in order to apply the high-order tangent plane projection (TNX) corrections. The bad pixel mask undergoes

the same transformation as the image. This task is run on each chip individually because "mscimage" will not apply TNX corrections on mosaicked images (this is a known IRAF issue).

The chips are combined into a composite Multi-extension FITS (MEF) file and "mscimage" is run a second time to create a single file with a single bad pixel mask including cosmic ray, bad column and chip gap locations. A catalogue of stellar positions is created using the IRAF task "mscred.mscgetcat". Stars falling anywhere within roughly 3500 pixels from the center and with magnitudes ranging from 19 to 10 are used. The IRAF task "mscred.msccmatch" is used to match the stellar positions to pixel positions in order to determine the lower-order corrections for the mosaicked image. These corrections are different from exposure to exposure due to various atmospheric effects and pointing errors. These small corrections are also applied to the bad pixel masks. Using the catalogue of stellar positions, a catalogue of PSFs are made for each FOV. This is used in the combining stage when PSF matching is required (next section).

\subsubsection{PSF-Matching and Stacking}

Before combining dithered exposures into a single, deep image, the various image groups are PSF-matched and aligned as follows. Using the PSF catalogues produced by "imacsreg", the mode seeing value is computed for each exposure and inserted into the header. The images are aligned based on the WCS information. These offsets account for any dithering or pointing errors. Bad pixel masks are also shifted accordingly. All similar frames are degraded to the worst seeing using the IRAF task "psfmatch" (making sure to remove frames with 
anomalously bad seeing). Similar frames are stacked using the "imcombine" task in IRAF. The process of combining images ignores masked pixels and also rejects all values above a certain threshold (e.g. 2 sigmas), thus removing any residual cosmic rays.

\section{Lessons learned}

The commissioning and implementation of MMTF as a facility instrument have been harder than anticipated. We highlight here the main lessons learned along the way.

\subsection{Technical Issues}

1. The etalon alignment depends on position angle. Detailed testing of MMTF revealed that the alignment of the MMTF etalon depended sensitively $( \pm \sim 0.03 \mu \mathrm{m}$; Figure 8$)$ on the rotation angle of the etalon with respect to the (horizontal) optical axis of the Nasmyth focus. This effect is not seen when the optical axis is near vertical, e.g. at Cassegrain focus. This effect is largely reproducible with position angle (Figure 8) and is attributed to the sagging of the large heavy plates of the MMTF etalon under gravity. It is now dealt with by forcing IMACS (and thus MMTF) to return to roughly the same gravity angle for each observation. This observing strategy has eliminated the need for time-consuming realignment and reduced the drift in the wavelength solution. However, it causes the field to rotate with respect to the CCD axes from observation to observation, so data reduction has to de-rotate the field before stacking a sequence of observations of the same target. We have found that this procedure does not affect the image quality of the final stack to within a fraction of a pixel even at the outer edge of the field.

2. The etalon alignment depends on $\lambda$. We also discovered that the $(X, Y)$ settings needed to align the etalon plates for a given etalon rotation angle were significantly different at longer wavelength (Figure 7). This means that the effective reflecting surfaces at long wavelengths are wedged with respect to the surfaces at shorter wavelengths, and vice-versa. We were expecting the effective gap (measured from $l=\lambda^{2} /[2 F S R]$ ) to vary with wavelength for a fixed gap (and this has been shown to be the case; Table 1; see also Rangwala et al. 2008), but we were not expecting the relative angle between the effective reflecting surfaces to vary this much with wavelength. This effect was dealt with by simply re-aligning the etalon plates after each change of order-blocking filter.

3. The etalon alignment does not depend on temperature. The CS100 X, Y settings needed to align the etalon plates were found to be very stable from run to run over a period of 
three years, given the same cables, CS100, etalon rotation angle, and wavelength were used. This implies that the etalon alignment is not sensitive to temperature changes. This reduces overheads associated with the installation and startup of MMTF.

4. The position of optical axis depends on etalon alignment. We have found that it is important to align the etalon plates before deriving the position of the optical axis.

5. The connectors to the cables are "fickle". MMTF uses a set of 15, 3, and $1 \mathrm{~m}$ cables provided by IC Optical Systems to connect the etalon to the CS100 controller. These long cables were covered by a copper sheath to shield them from the telescope electronic noise. These cables need to be handled with care. This is particularly true of the connectors which have a tendency to loosen easily. Applying a small drop of screw locking compound or cyanoacrylate glue (super glue) is often a good cure for this problem.

6. The focal length of the $f / 2$ camera depends on $\lambda$ and $Z_{\text {coarse }}$. The empirically derived focal length of the $\mathrm{f} / 2$ camera was found to depend slightly on wavelength and coarse etalon spacing $\left(Z_{\text {coarse }}\right)$ (see Figure 12$)$. The wavelength dependence indicates slight chromatic aberrations due to IMACS, MMTF, or both. The change in focal length is steeper beyong $\sim 6600 \AA$, roughly coincident with the change in etalon alignment (Section 5.1.2, Figure 7). We therefore speculate that MMTF (more specifically its coatings) is at least partly responsible for the chromatic aberrations. The dependence of the focal length on etalon spacing (at fixed wavelength) adds support to this idea. We calibrated these dependences and modified the wavelength calculator to take this effect into account.

7. The CS/FS mode of MMTF is of specialized use. The "active area" of the detector in the $\mathrm{CS} / \mathrm{FS}$ mode is at $7^{\prime}$ or more from the optical axis so the monochromatic area in this mode is a ring with only $F W H M \approx 2 ! 1$ or less. This mode is therefore only good for relatively small objects and does not take advantage of the large FOV of MMTF.

8. The full-field finesse of the MMTF etalon is lower than expected. Irregularities in the plate surfaces and coating thicknesses, as well as deviations from perfectly parallel plates, degrade the effective finesse (hence the efficiency of observing), from a value of $~ 50$ expected from the reflectivity curve (Figure 3 ) to a value of $\sim 24-29$ (Table 1 ).

\subsection{Scientific Issues}

\subsubsection{Strengths of $M M T F$}

Our experience has helped us appreciate the scientific strengths and weaknesses of MMTF. One outstanding feature of MMTF is its very large FOV ( $27^{\prime}$ diameter), making it 
an ideal survey instrument, particularly if monochromaticity is not a critical factor. And, even if it is, the large Jacquinot spot (up to $\sim 13^{\prime}$ in low resolution mode; Table 2) of MMTF makes it a very powerful instrument which achieves a significant efficiency advantage over conventional long-slit spectrographs (Jacquinot 1954).

The large aperture of the Magellan-Baade telescope, superb delivered image quality (DIQ $\sim 0^{\prime \prime} .5$ ) of the IMACS $\mathrm{f} / 2$ camera, excellent photometric characteristics of the Las Campanas site, and ability of MMTF to tune the transmitted wavelength to any feature within the range $\sim 5000-9200 \AA$ combine to provide excellent emission-line sensitivity to point sources or filamentary structures (see Table 3) and make MMTF a highly versatile instrument.

Through rejection of significant sky and/or continuum emission (compared to conventional narrow-band filters), MMTF can significantly improve observing efficiency for reaching a desired signal-to-noise ratio. The gain is particularly high in spectral regions where $\mathrm{OH}$ sky lines are densely distributed $(\gtrsim 7000 \AA)$.

MMTF can switch between two wavelengths on short timescales (e.g. on-band and offband images obtained in sequence or nearly simultaneously through the use of the CS/FS mode). This allows the user to correct for time-varying observational effects, including atmospheric transmission and sky brightness. It also allows for differential imaging on short timescales.

FP tunable filters in general are unbeatable for wide-field diffuse light detection (no IFUs can ever compete in this area of research), although this type of program does not take advantage of the excellent DIQ of MMTF/IMACS.

\subsubsection{Weaknesses of $M M T F$}

Contrary to TTF (e.g. Bland-Hawthorn \& Kedziora-Chudczer 2003), MMTF has proven difficult to use in bright moon conditions. Scattered light in the $\mathrm{f} / 2$ camera and MMTF optics

makes data obtained in these conditions tricky to reduce. Observations under grey or darker conditions do not show these problems.

Internal scattering also produces complex halos around bright stars. The shape of the halos and their integrated intensity relative to that of the star $(\sim 10 \%)$ vary across the field so they are difficult to model. Targets near very bright stars have been observed by putting the star within the chip gap.

MMTF is not a good instrument for programs requiring multiple $(>2)$ blocking filter 
switches in one night. Each filter used requires a set of careful wavelength calibration during the day ( $\sim 1$ hour each; see Section 4.4.2 for more detail). Changing filters in the middle of the night adds an overhead of 15-30 minutes due to these calibrations.

MMTF is better suited for deep observations of a few targets per night rather than snapshots of a large number of targets due to the overhead associated with the rotation of IMACS for each target.

\section{Outlook}

MMTF is currently used at a rate of $\sim 10$ nights per semester. As MMTF becomes a facility instrument and a community of users is created, we anticipate that this rate will increase.

To our knowledge, two similar instruments on 10-meter class telescopes will soon be in routine operation: the imaging Fabry-Perot system for the Robert Stobie Spectrograph on the South African Large Telescope (Rangwala et al. 2008) and OSIRIS, the Optical System for Imaging and Low-Intermediate-Resolution Integrated Spectroscopy on the Gran Telescopio Canarias (Cepa et al. 2007 and references therein). These instruments will cover a different area of the parameter space than MMTF, taking advantage of the larger collecting area of their host telescopes but over a smaller FOV than MMTF. As they mature, the combination of these three tunable filters will provide formidable tools to study emissionline systems, near and far. MMTF will remain the widest field device of its kind in the foreseeable future.

S.V., B.J.W., D.S.N.R., and M.M. were supported in part by NSF through contracts AST/ATI 0242860 and AST/EXC 0606932. S.V. acknowledges support from a Senior Award from the Alexander von Humboldt Foundation and thanks the host institution, MPE Garching, where this paper was written. The MMTF team is grateful for the support provided for this project by the past and present Directors of the Observatories of the Carnegie Institution for Science, Gus Oemler and Wendy Freedman, the Magellan Technical Manager, Alan Uomoto, the Magellan Council, and the technical staff at the Las Campanas Observatory. We also thank P.L. Shopbell and A. Bagish who helped in the early stages of the MMTF testing and commissioning, and M. Bergamo who helped put together the GUI for the MMTF Exposure Time Calculator. Last but not least, we are very grateful to R.J. Weymann who, more than eight years ago, helped initiate this project which is now a reality. 


\section{REFERENCES}

Atherton, P.D. et al. 1981, Opt. Eng., 20, 806

Bland, J., \& Tully, R. B. 1989, AJ, 98, 723

Bland-Hawthorn, J. 2000a, in Imaging the Universe in Three Dimensions. Proc. ASP Conf. Vol. 195. Edited by W. van Breugel and J. Bland-Hawthorn, p. 34

Bland-Hawthorn, J. 2000b, in Encyclopedia of Astronomy \& Astrophysics, MacMillan and Institute of Physics Publishing.

Bland-Hawthorn, J., \& Jones, D. H. 1998a, PASA, 15, 44

Bland-Hawthorn, J., \& Jones, D. H. 1998b, SPIE, 3355, 855

Bland-Hawthorn, J., \& Kedziora-Chudczer, L. 2003, PASA, 20, 242

Cepa, J., et al. 2007, RMxAC, 29, 168

Dopita, M. A., \& Hua, C. T. 1997, ApJS, 108, 515

Dressler, A., Hare, T., Bigelow, B. C., \& Osip, D. J. 2006, SPIE, 6269, 62690F

Hamuy, M. et al. 1992, PASP, 104, 533

Hamuy, M. et al. 1994, PASP, 106, 566

Jacquinot, P. 1954, J Opt Sci Amer, 44, 761

Jones, D. H., \& Bland-Hawthorn, J. 1998, PASP, 110, 1059

Jones, D. H., Shopbell, P. L., \& Bland-Hawthorn, J. 2002, MNRAS, 329, 759

Macleod, H. A. 2001, "Thin-Film Optical Filters", CRC Press, 3rd edition, 641 p.

Oke, J. B. 1990, AJ, 99, 1621

Rangwala, N., Williams, T. B., Pietraszewski, C., \& Joseph, C. L. 2008, AJ, 135, 1825 
Table 1. Characteristics of the ET-150 Etalon in MMTF

\begin{tabular}{|c|c|c|c|c|c|}
\hline $\begin{array}{l}Z_{\text {coarse }} \\
\quad(1)\end{array}$ & $\begin{array}{l}m \\
(2)\end{array}$ & $\begin{array}{c}l(\mu \mathrm{m}) \\
(3)\end{array}$ & $\begin{array}{c}F W H M(\AA) \\
(4)\end{array}$ & $\begin{array}{c}F S R(\AA) \\
\quad(5)\end{array}$ & $\begin{array}{l}N \\
(6)\end{array}$ \\
\hline \multicolumn{6}{|c|}{$\lambda=5102 \AA$} \\
\hline-2 & 27.5 & 7.0 & 13.7 & 186 & 13.6 \\
\hline+1 & 37.7 & 9.6 & 10.1 & 135 & 13.4 \\
\hline+3 & 47.6 & 12.1 & 8.0 & 107 & 13.4 \\
\hline \multicolumn{6}{|c|}{$\lambda=6600 \AA$} \\
\hline-2 & 20.0 & 6.6 & 11.8 & 314 & 26.6 \\
\hline+1 & 29.0 & 9.6 & 8.1 & 219 & 27.0 \\
\hline+3 & 35.0 & 11.6 & 6.6 & 181 & 27.4 \\
\hline \multicolumn{6}{|c|}{$\lambda=7045 \AA$} \\
\hline-2 & 22.8 & 8.3 & 10.3 & 299 & 29.0 \\
\hline+1 & 30.8 & 11.1 & 7.7 & 224 & 29.1 \\
\hline+3 & 35.8 & 13.0 & 6.6 & 191 & 28.9 \\
\hline \multicolumn{6}{|c|}{$\lambda=9163 \AA$} \\
\hline-2 & 22.0 & 10.2 & 17.2 & 414 & 24.1 \\
\hline+1 & 29.0 & 13.5 & 13.1 & 314 & 24.0 \\
\hline+3 & 33.0 & 15.3 & 11.5 & 276 & 24.0 \\
\hline
\end{tabular}

Note. - Col. (1): Coarse $Z$ setting on etalon. Col. (2): Order number. Col. (3): Plate spacing in $\mu \mathrm{m}$. Col. (4): Spectral resolution in $\AA$. Col. (5): Free spectral range in $\AA$. Col. (6): Effective finesse $=F S R / F W H M$.

\section{Table 2. Jacquinot Spot Diameters}

\begin{tabular}{ccc}
\hline \hline $\begin{array}{c}\lambda(\AA) \\
(1)\end{array}$ & $D_{J}($ Zcoarse $=-2)(\operatorname{arcmin})$ & $D_{J}($ Zcoarse $=+3)(\operatorname{arcmin})$ \\
\hline 5102 & $(2)$ & $(3)$ \\
6600 & $13.8^{\mathrm{a}}$ & $10.7^{\mathrm{a}}$ \\
9163 & 11.6 & 8.5 \\
& 11.5 & 9.5 \\
\hline
\end{tabular}

Note. - Col. (1): Central wavelength. Col. (2): Jacquinot spot diameter at Zcoarse $=-2$. Col. (3): Jacquinot spot diameter at Zcoarse $=+3$.

${ }^{\mathrm{a}}$ At $5100 \AA$ the numbers are $\sim 1-2^{\prime}$ higher due to a drop in finesse. 
Table 3. MMTF Detectable Flux and Throughput

\begin{tabular}{|c|c|c|c|c|}
\hline $\begin{array}{c}\lambda \\
(\AA) \\
(1)\end{array}$ & $\begin{array}{c}\text { Detectable Flux } \\
\left(10^{-17} \mathrm{erg} \mathrm{s}^{-1} \mathrm{~cm}^{-2}\right) \\
(2)\end{array}$ & $\begin{array}{c}\text { Detectable Flux } \\
\left(10^{-17} \mathrm{erg} \mathrm{s}^{-1} \mathrm{~cm}^{-2}\right) \\
(3)\end{array}$ & $\begin{array}{c}\text { Detectable Flux } \\
\left(10^{-17} \mathrm{erg} \mathrm{s}^{-1} \mathrm{~cm}^{-2}\right) \\
(4)\end{array}$ & $\begin{array}{c}\text { Throughput } \\
\left(10^{16} \text { counts } \mathrm{erg}^{-1} \mathrm{~cm}^{2}\right) \\
(5)\end{array}$ \\
\hline 5102 & 2.90 & 3.27 & 4.44 & 0.11 \\
\hline 6600 & 0.66 & 0.75 & 1.04 & 1.42 \\
\hline 6815 & 0.40 & 0.45 & 0.61 & 1.76 \\
\hline 7045 & 0.52 & 0.59 & 0.78 & 1.42 \\
\hline 8149 & 1.98 & 2.09 & 2.47 & 0.18 \\
\hline
\end{tabular}

Note. - Col. (1): Wavelength. Col. (2): Flux of a 5- $\sigma$ emission-line point source in a 1-hour exposure, assuming Moon age $=0$ days, $0.5^{\prime \prime}$ seeing, $1^{\prime \prime}$ diameter extraction aperture, and airmass of 1.1. Col. (3): Same as Col. (2) but Moon age $= \pm 7$ days. Col. (4): Same as Col. (2) but Moon age $=14$ days. Col. (5): Ratio of count rate to flux. 


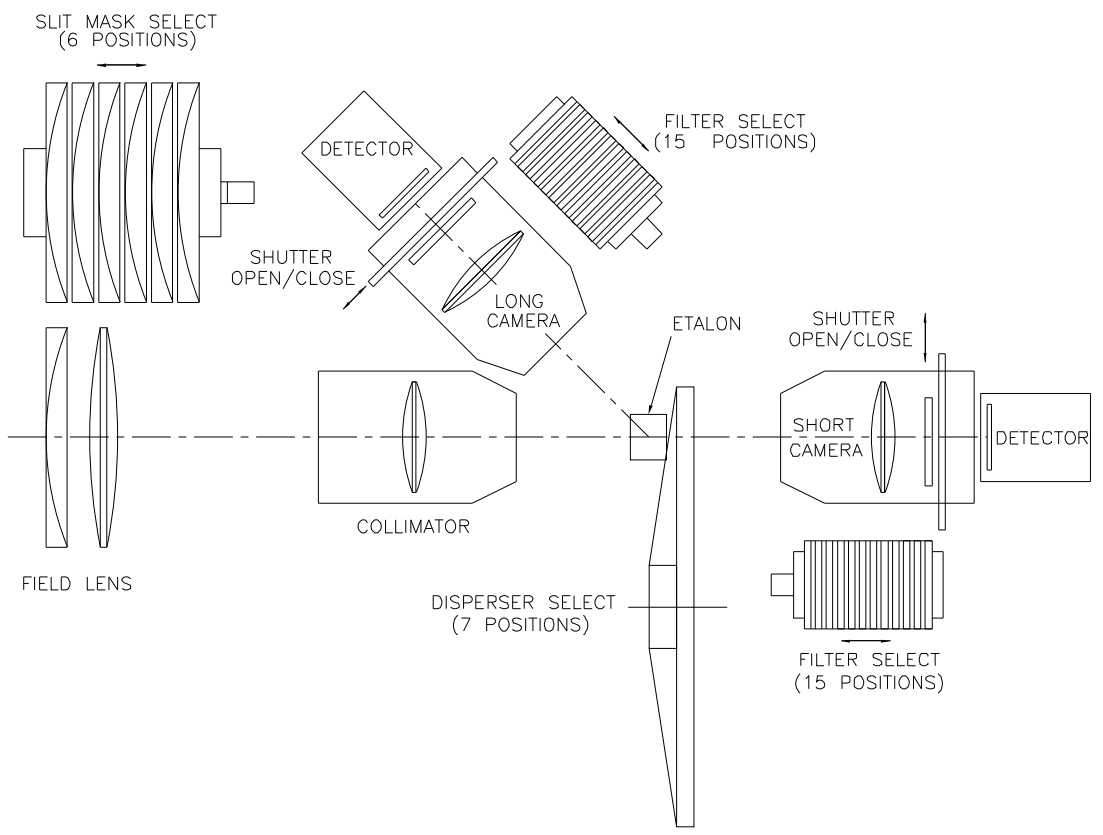

Fig. 1. - The optical path through IMACS and the MMTF. The MMTF etalon is placed in the collimated beam of the $\mathrm{f} / 2$ camera between the collimator and the camera. 


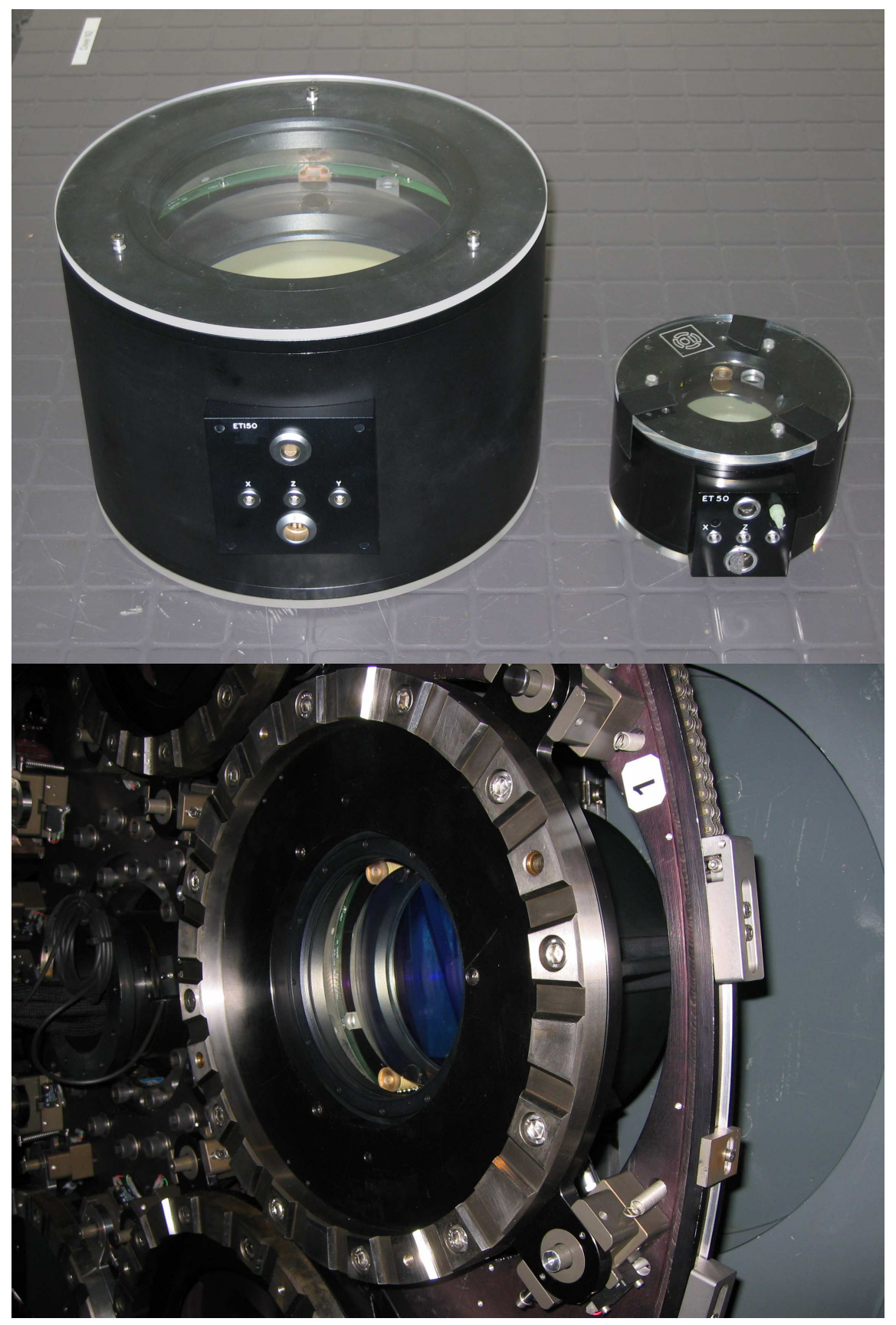

Fig. 2.- (Top) The ET150 etalon used for MMTF alongside the smaller ET50 etalon for comparison. (Bottom) The MMTF ET150 etalon mounted in the IMACS disperser wheel. 


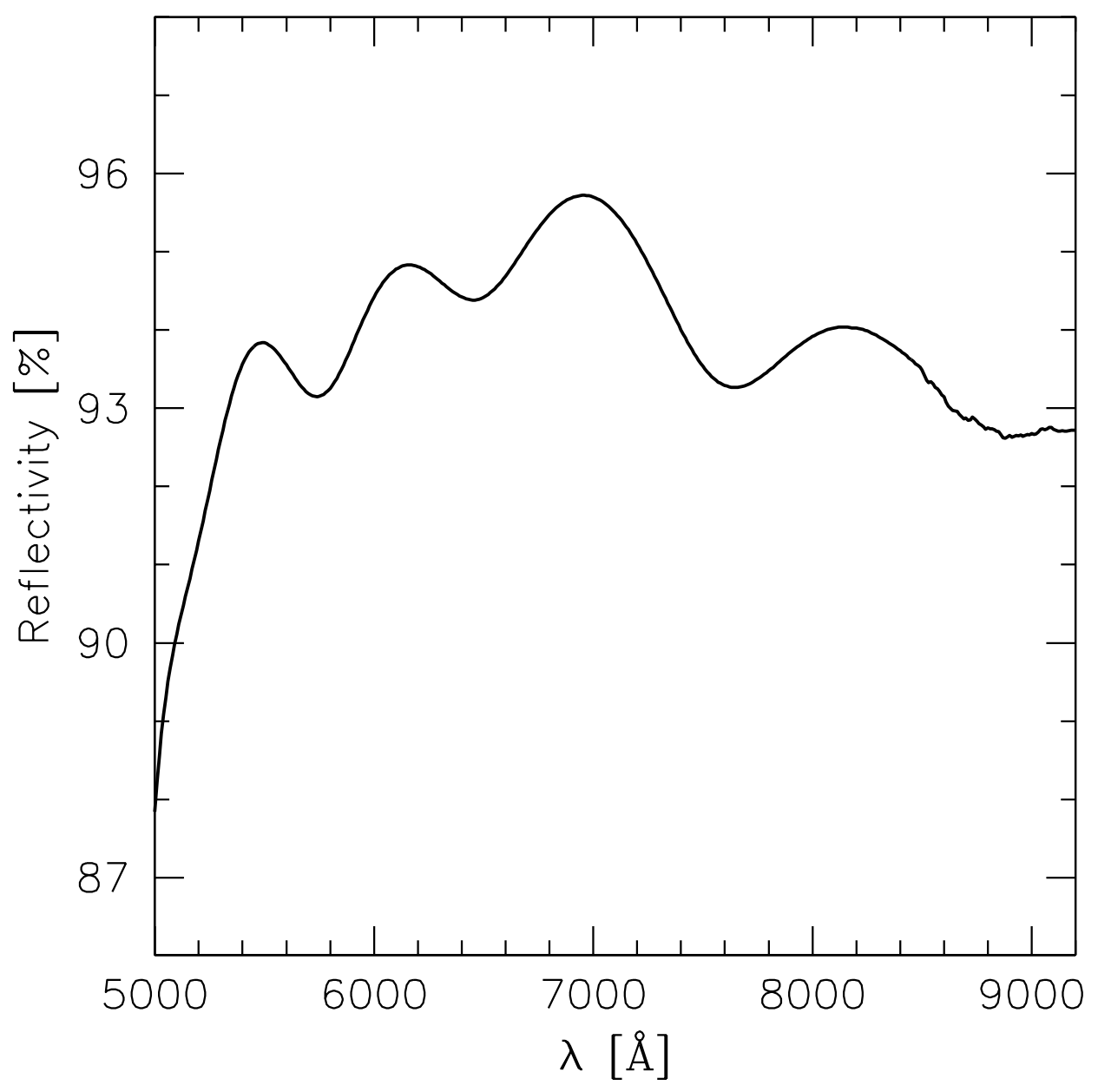

Fig. 3.- The reflectivity curve of the MMTF ET150 etalon coating. 


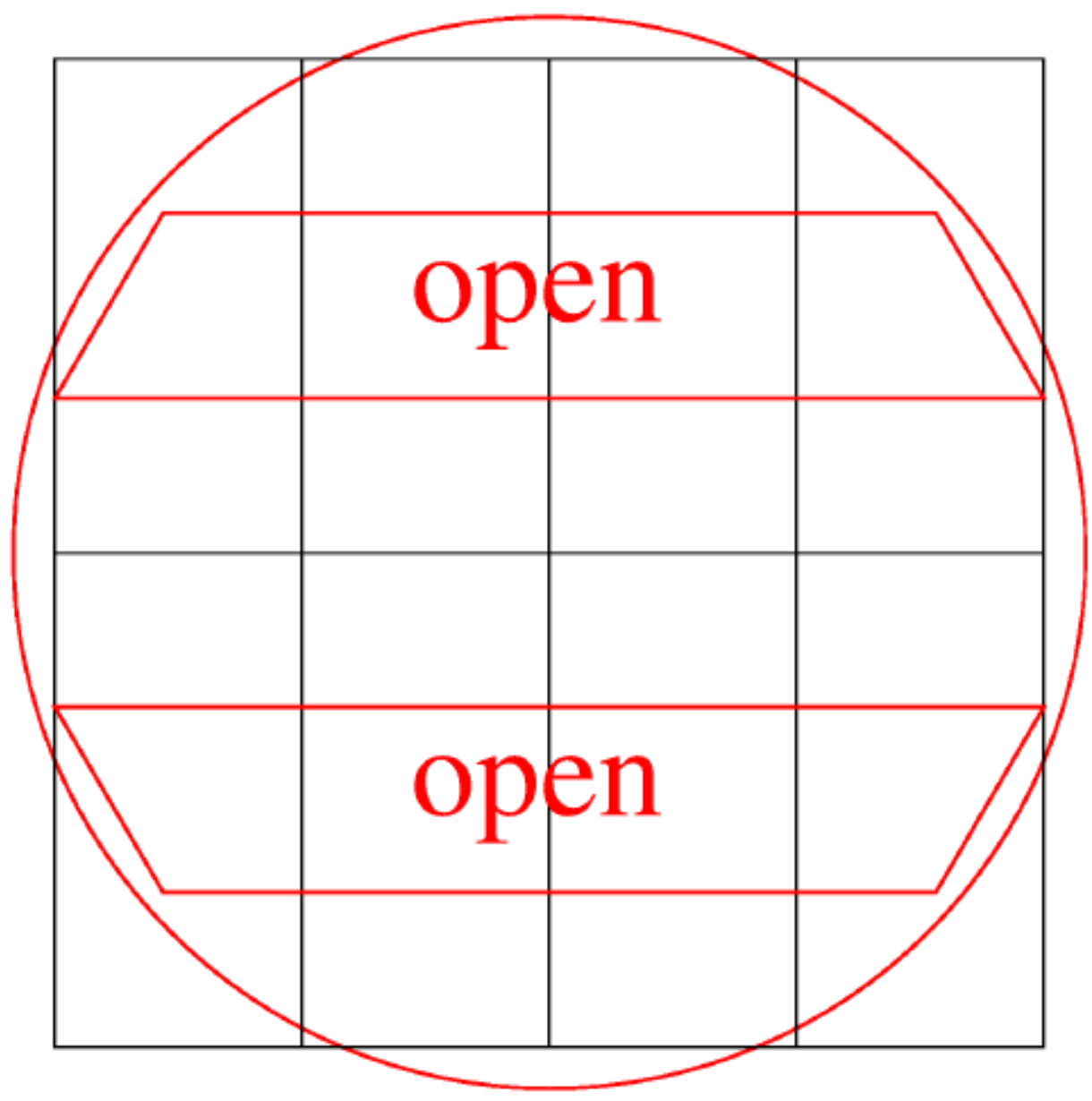

Fig. 4. - Diagram of the aperture mask used in charge shuffling / frequency switching mode. The black outline shows the 8 CCD chips, and the red outline the aperture mask. The "open" segments are illuminated. 


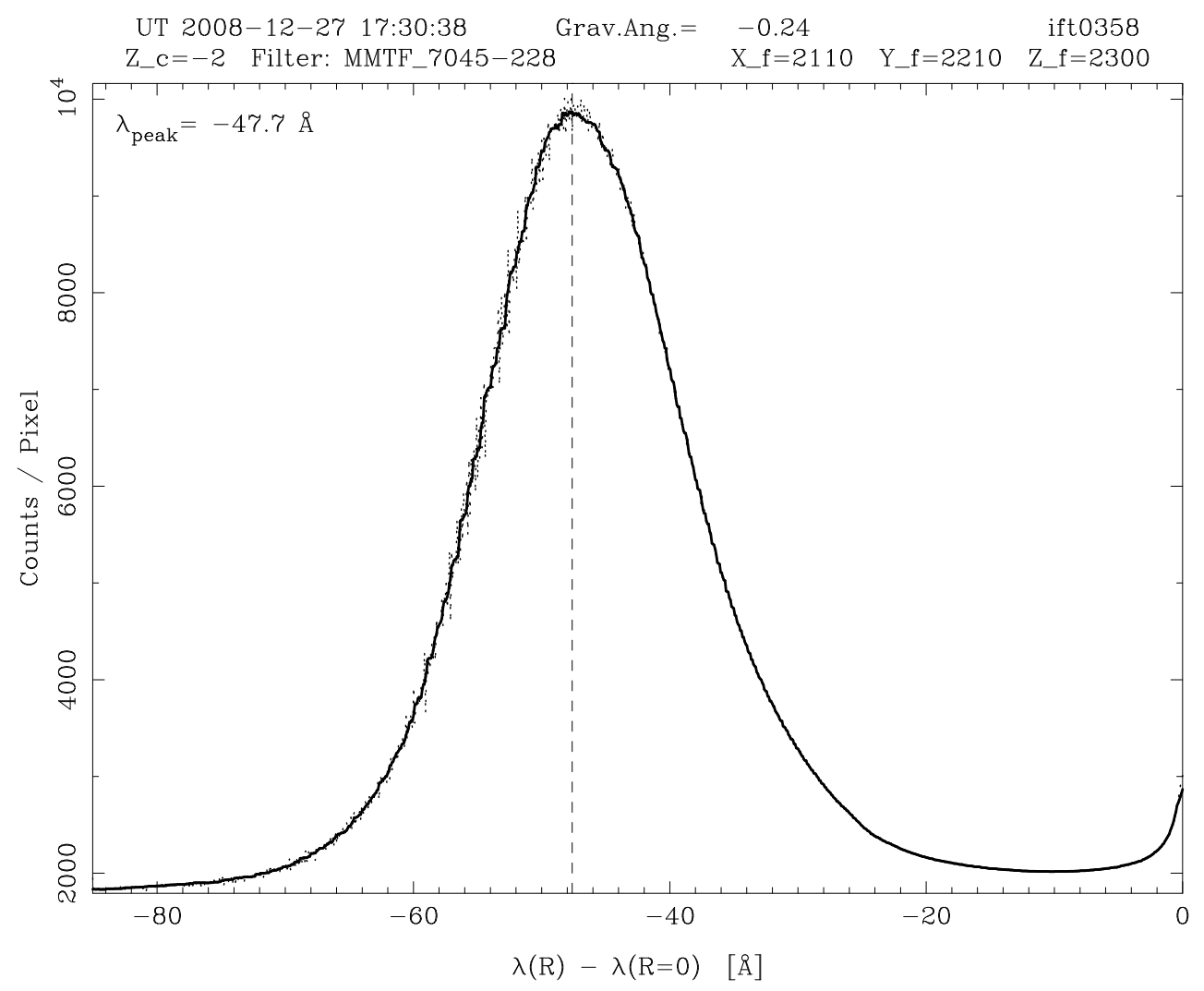

Fig. 5.- Emission-line spectrum derived by azimuthally averaging reference rings from an arc lamp (Argon in this case). The dotted line is the azimuthally averaged data, while the solid line is the data median smoothed by a $1 \AA$ window. Reference rings are taken at regular intervals to monitor wavelength drift and plate alignment during the night (see Figures 6 and 11). 


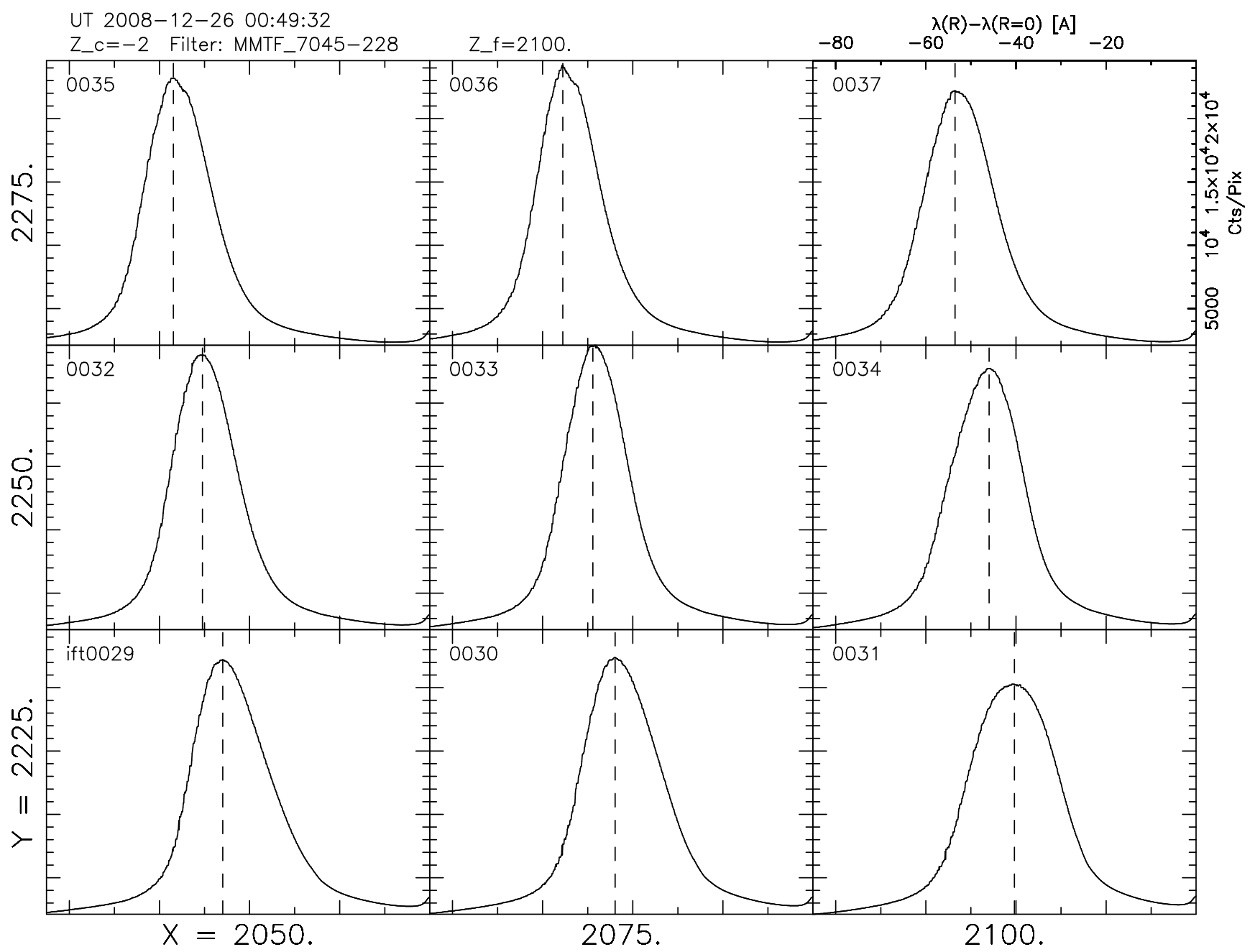

Fig. 6.- Typical output from the plate alignment procedure. Reference rings are taken over a range in $X$ and $Y$. The profiles of emission features are compared by eye (and, where possible, with line fits) to find the narrowest and most symmetric profile (in this case, exposure \#033 in the center with $X \simeq 2075$ and $Y \simeq 2250$ ). 


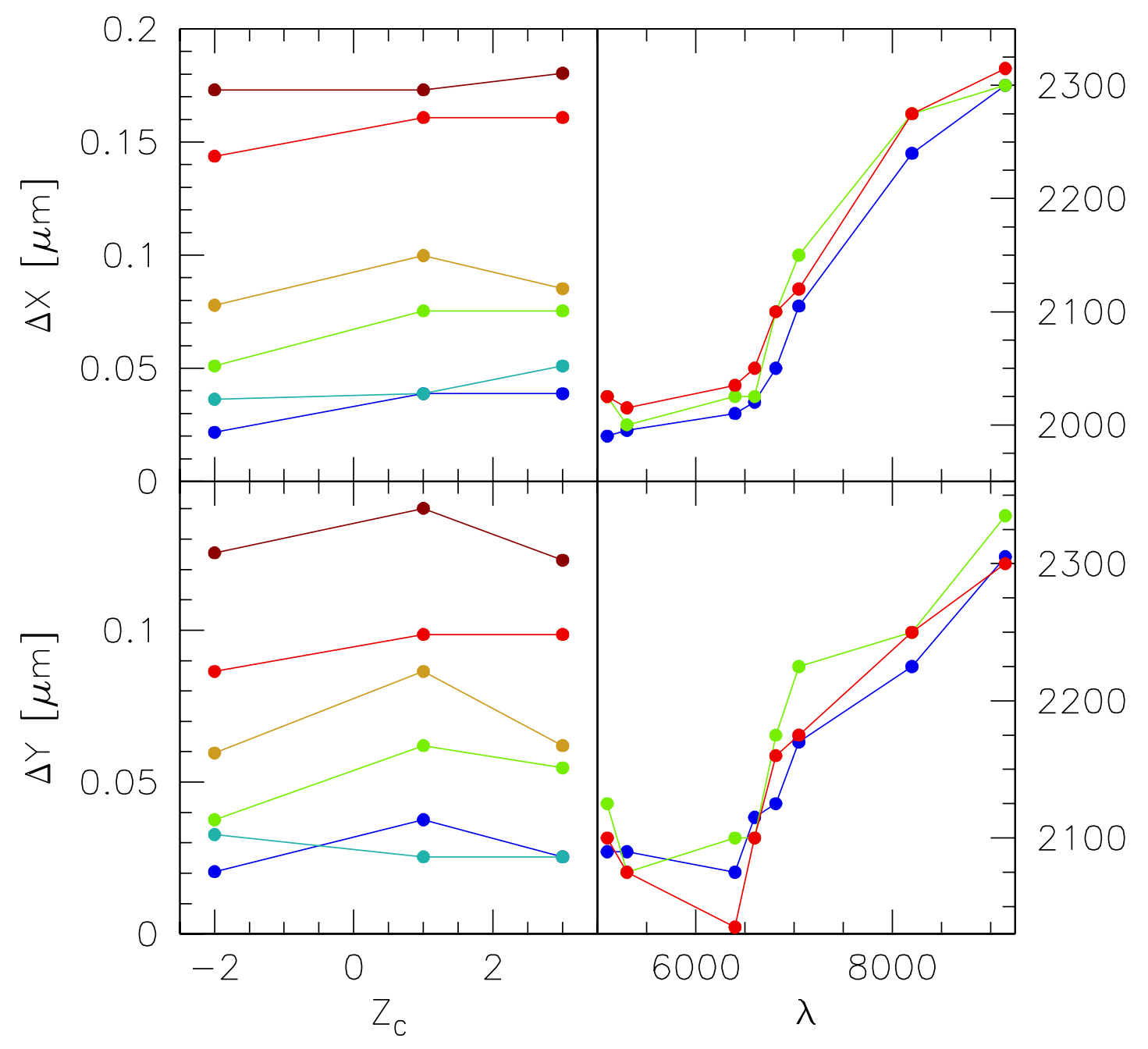

Fig. 7.- Dependence of etalon alignment on etalon gap and wavelength, for a given etalon rotation angle. Shown on these plots are either the difference in $X$ and $Y$ positions in $\mu \mathrm{m}$ (on the left vertical axis) or in IMACS/MMTF computer control software units (on the right vertical axis). In the left panels, $\mathrm{Z}_{C}$ is the coarse gap spacing, while the different colors represent the wavelength: $5100 \AA$ (blue), $6400 \AA$ (turquoise), $6800 \AA$ (green), 7050 $\AA$ (orange), $8150 \AA$ (red) and $9150 \AA$ (deep red). In the right panels, the colors represent the coarse gap spacing: $\mathrm{Z}_{C}=-2$ (blue), +1 (green) and +3 (red). Note the change in $\mathrm{X}$ and $\mathrm{Y}$ of $\sim 0.10-0.15 \mu \mathrm{m}$ for $\lambda \gtrsim 6600 \AA$. This indicates that the relative angle between the effective reflecting surfaces of the etalon varies with wavelength. 


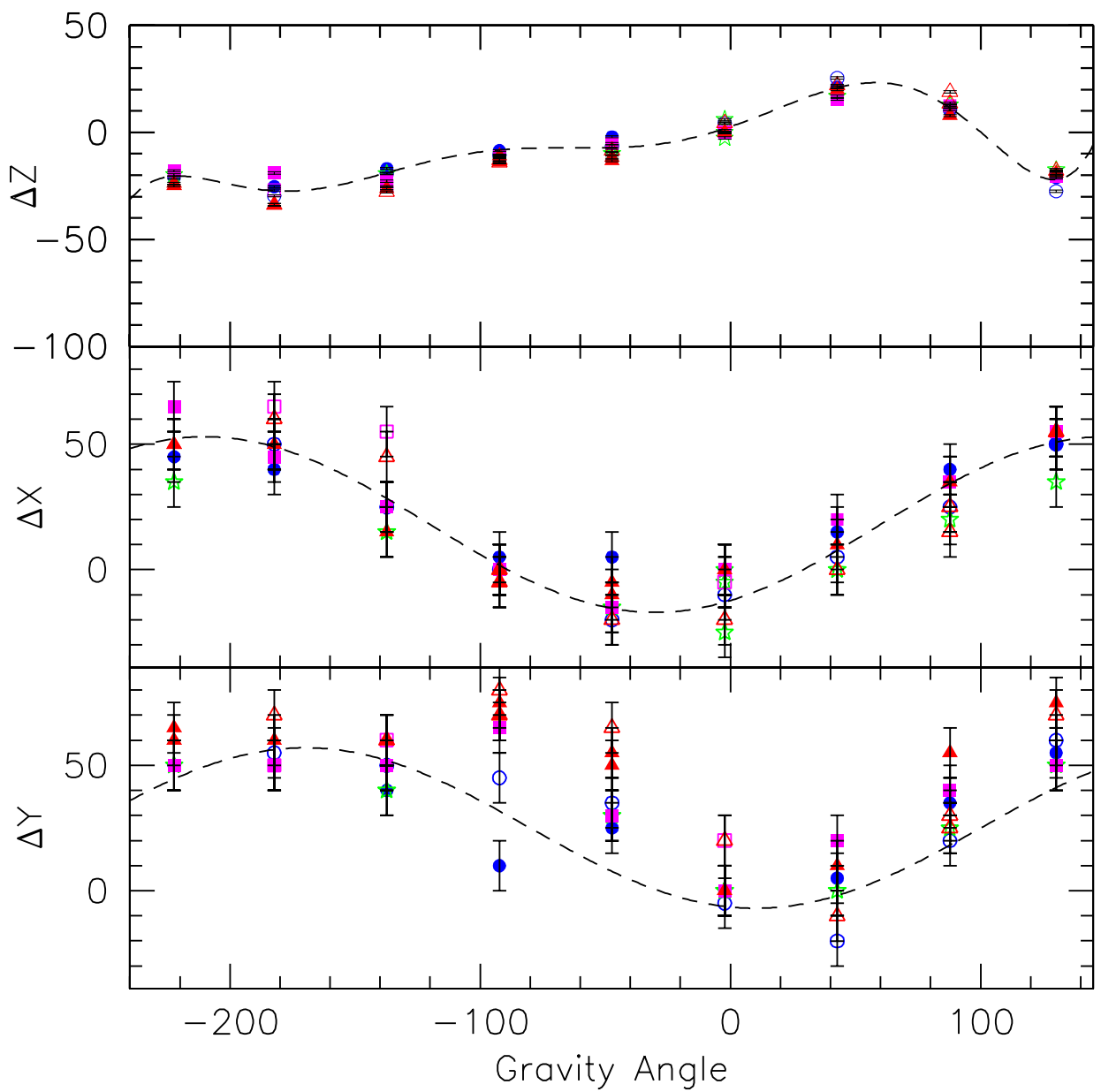

Fig. 8.- Dependence of etalon alignment on gravity angle for a coarse gap spacing of $\mathrm{Z}_{c}=$ -2 . The total amplitude of the change in alignment with gravity angle is $\sim 60$ in IMACS computer control software units or $\sim 0.03 \mu \mathrm{m}$, but is generally highly reproducible (for reasons which remain unclear, the alignment values become difficult to predict for gravity angles between -120 and -20 degrees). The different point types and colors refer to sequences taken together. 


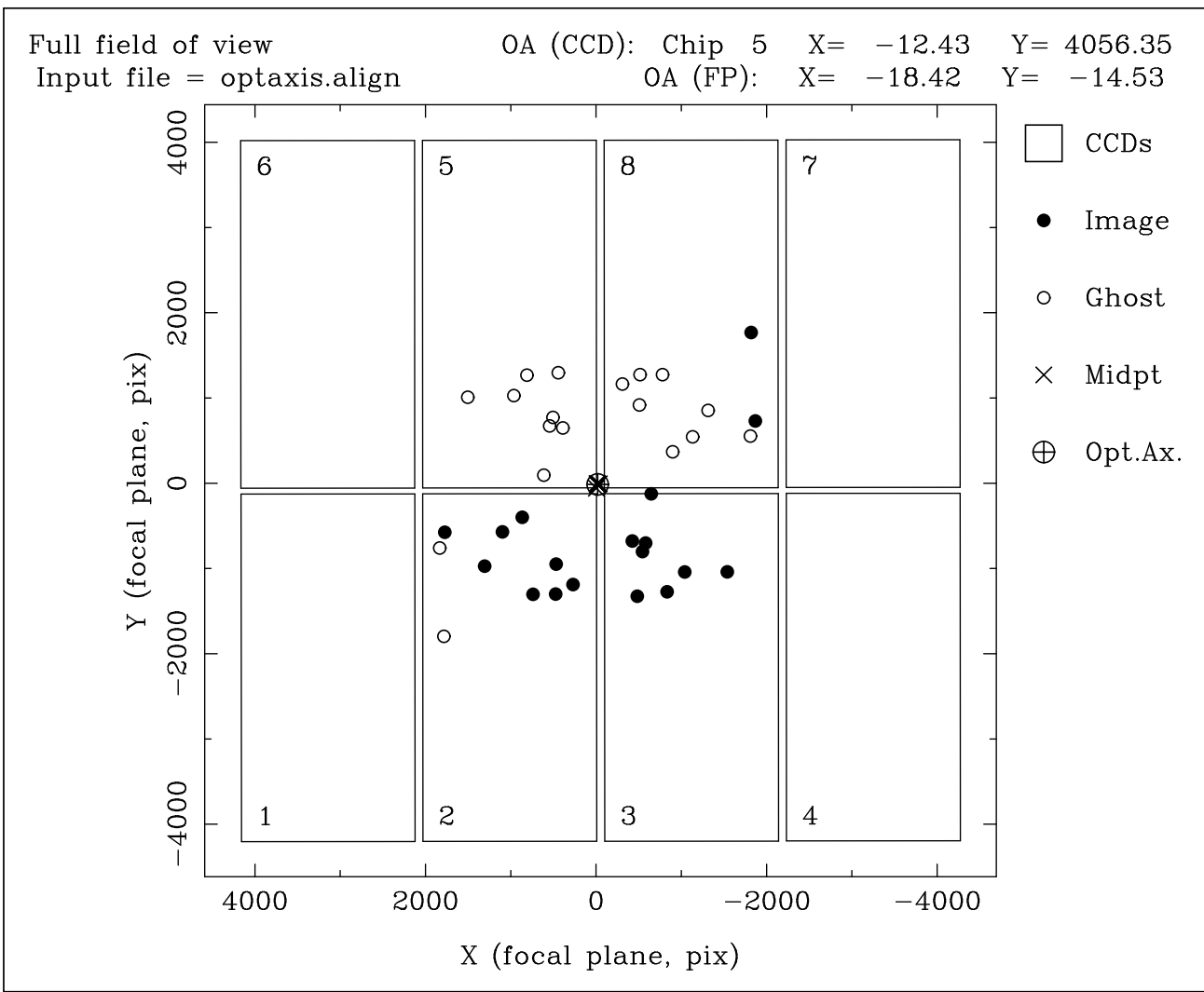

Fig. 9. - Example of output from the procedure used to find the position of the optical axis. The position is typically accurate to \pm 1 pixel $(0,2)$. 


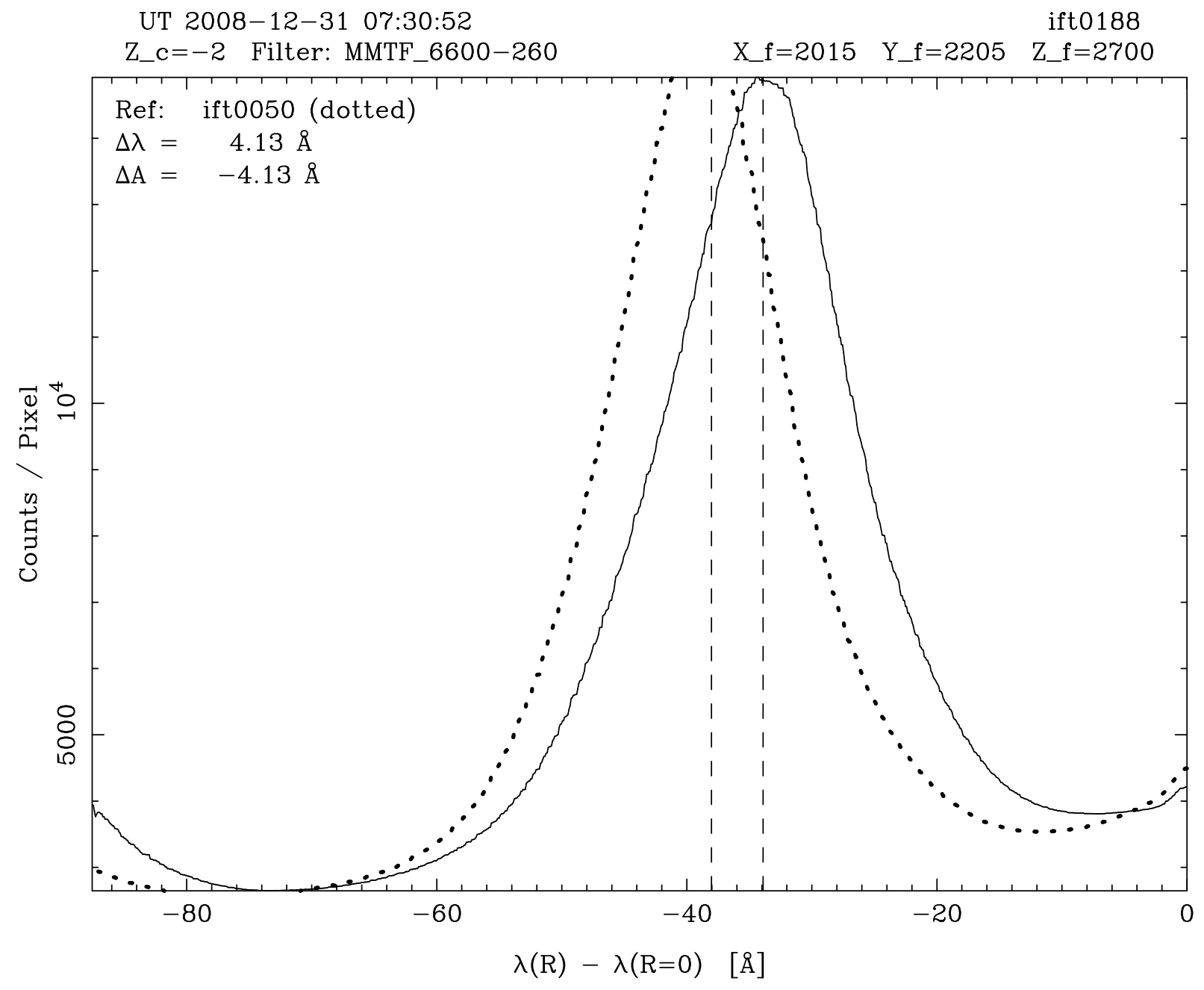

Fig. 10.- Procedure to monitor wavelength drift. The emission-line profiles derived from reference rings taken at different times are compared and the shift is used to update the wavelength solution. 


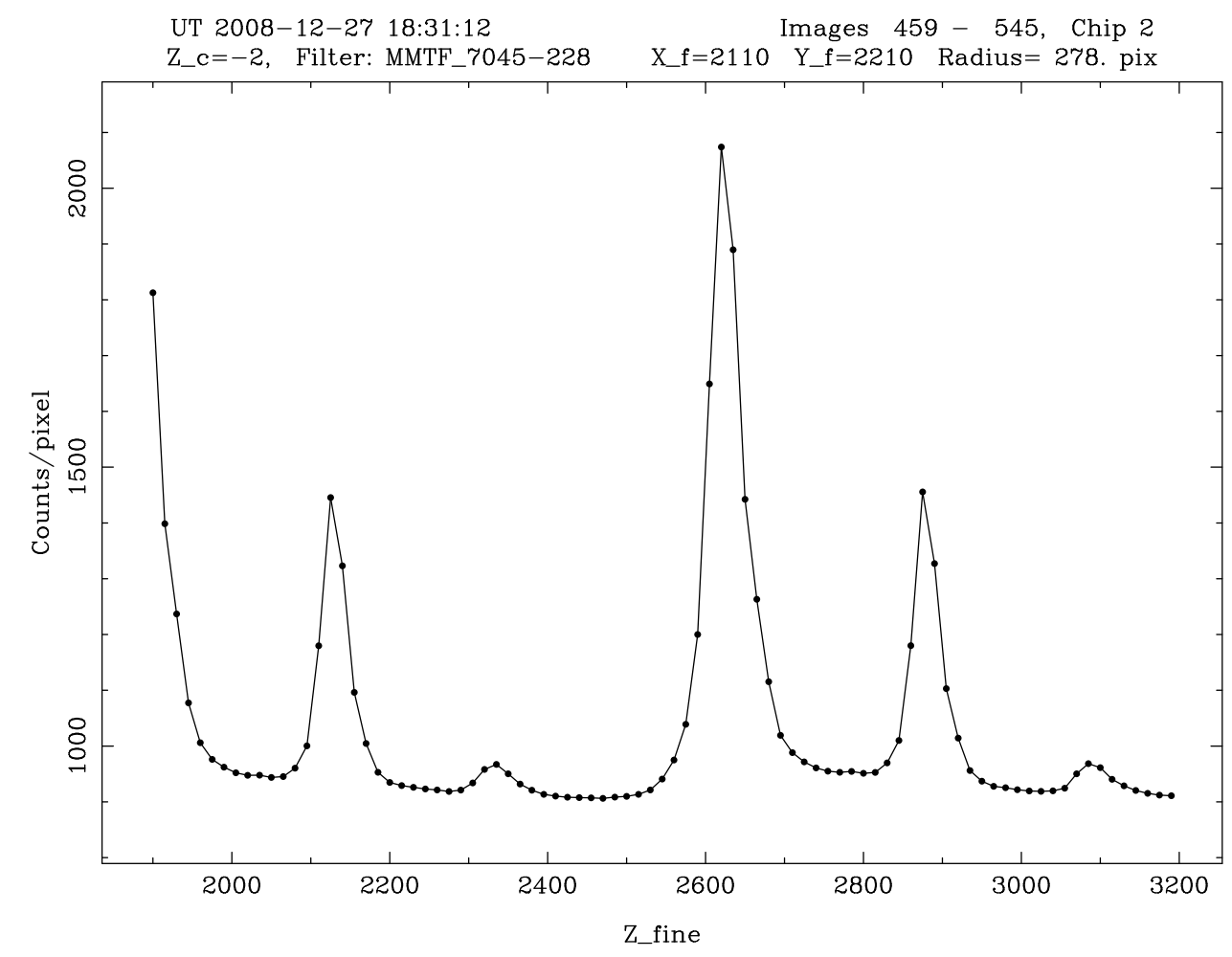

Fig. 11. - Spectrum extracted from a "data sausage" of Argon. The data sausage is obtained by stepping the etalon spacing in small increments (Nyquist-sampled) through at least one FSR, but reading out only a small CCD subraster. The lines at $Z_{\text {fine }} \sim 2120$ and 2330 correspond to Ar I $\lambda \lambda 7067.218,7147.042$, respectively. They are repeated at $Z_{\text {fine }} \sim 2885$ and 3095. The line at $Z_{\text {fine }} \sim 2615$ is $\operatorname{Ar}$ I $\lambda 6965.431$. This particular sausage was obtained 278 pixels from the optical axis. The wavelength calibration procedure simultaneously acquires eight data sausages like this one at eight different positions (distances) from the optical axis and use them to derive the full wavelength solution. 

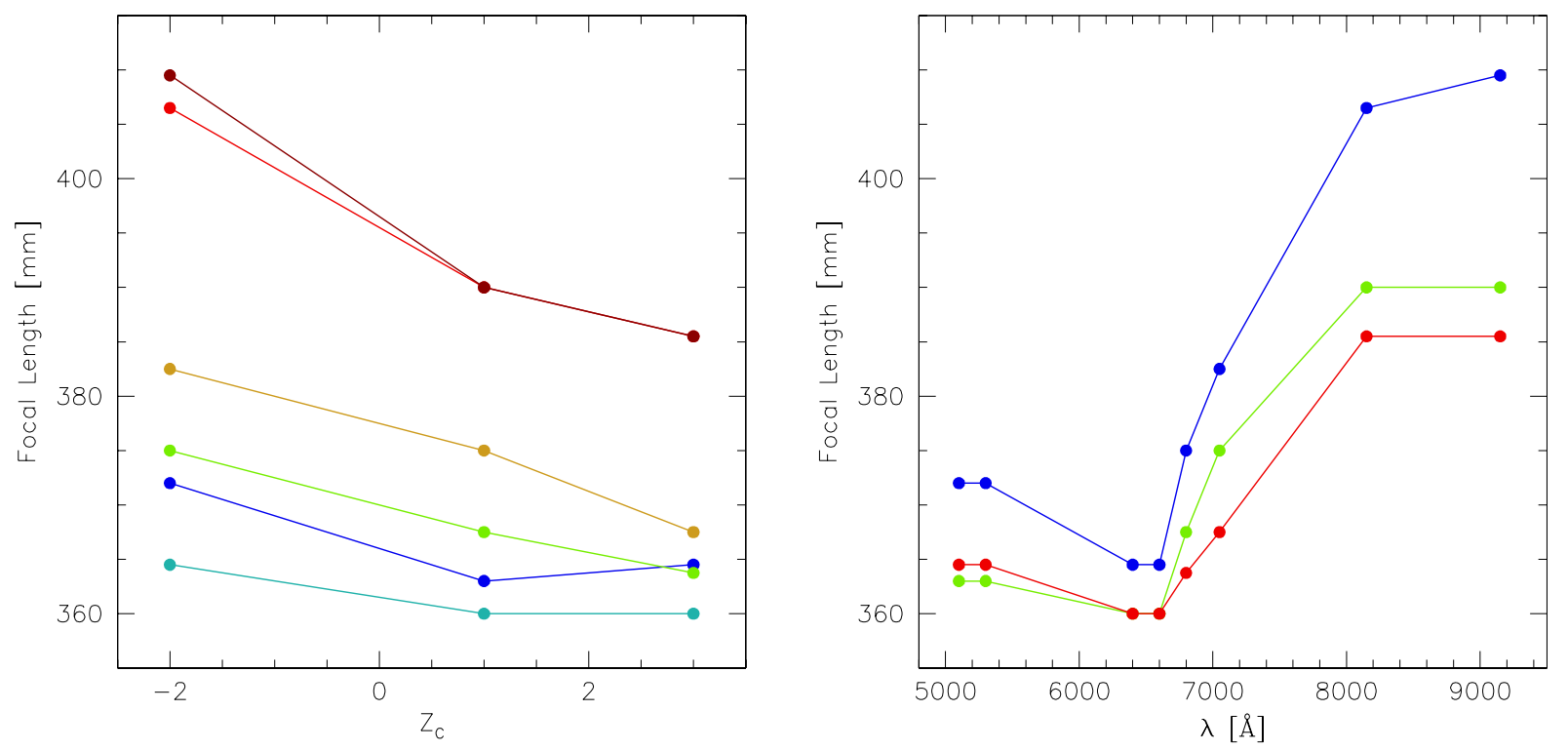

Fig. 12. - Dependence of the focal length (in $\mathrm{mm}$ ) on etalon gap and wavelength. In the left panel, $\mathrm{Z}_{C}$ is the coarse gap spacing, while the different colors represent the wavelength: $5100 \AA$ (blue), $6400 \AA$ (turquoise), $6800 \AA$ (green), $7050 \AA$ (orange), $8150 \AA$ (red) and 9150 $\AA$ (deep red). In the right panel, the colors refer to the coarse gap spacing: $Z_{C}=-2$ (blue), +1 (green) and +3 (red). These results suggest slight chromatic aberrations due in part to MMTF. 